\title{
Metabolite Fingerprinting Eleutherine palmifolia (L.) Merr. Using UPLC-QTOF-MS/MS
}

\author{
Roihatul Mutiah*, Weka Sidha Bhagawan, Burhan Ma'arif Zainal Arifin, Jauhar Maknun Septaza \\ Rahmandika
}

Department of Pharmacy, Faculty of Medical and Health Science, Maulana Malik Ibrahim State Islamic University of Malang

\begin{abstract}
Eleutherine palmifolia (L.) Merr. (E. palmifolia) is an Indonesian native plant that has the potential to be developed into phytopharmaca. The differences in growth locations are thought to cause variation in the content of metabolite compounds which affect differences in pharmacological activity. This study aims to determine the profile of metabolites E. palmifolia bulb from several regions in Indonesia. The samples were collected from six different locations, namely East Java, Central Java, West Java, East Borneo, Central Borneo, and South Borneo. Sample extraction was carried out using Ultrasonic Assisted Extraction (UAE) method with $96 \%$ ethanol. The analysis of the content of metabolites was carried out using UPLC-QTOF-MS/MS with a stationary phase column $\mathrm{C}_{18}$ (Okta Decyl Silica), mobile phase mixture of formic acid /water 0.1/99.9 $(\mathrm{v} / \mathrm{v})$, and formic acid/acetonitrile $0,1 / 99,9(\mathrm{v} / \mathrm{v})$. The results of the analysis were interpreted using software Masslynx and continued with chemometric analysis using the method Principle Component Analysis (PCA). The results showed that there were differences in the content of the metabolite compounds in $E$. palmifolia bulb originating from six different regions.
\end{abstract}

Keywords: Eleutherine palmifolia, Metabolite fingerprinting, UPLC-QTOF-MS/MS

\section{INTRODUCTION}

Metabolite fingerprinting is a fast and accurate method to determine the content of compounds contained in a plant extract (metabolome) using chromatographic techniques such as HighPerformance Thin Layer Chromatography (HPTLC), High-Performance Liquid Chromatography (HPLC), Gas chromatography (GC), and Mass spectrometry (MS) (Srivastava et al., 2010). The metabolite profiling technique can be combined with profiling bioassays such as cytotoxic tests, antimicrobial tests, then linked to the results of multivariate statistical analysis. Using these techniques, efforts to find new compounds can be done quickly and efficiently (Wolfendera et al., 2015).

Eleutherine palmifolia (L.) Merr., is one of the native plants of Indonesia, especially the island of Borneo, which has the potential to be developed into phytopharmaca. Epalmifolia bulb has long been used empirically for the treatment of several diseases. Similar to the onion group, E. palmifolia bulb is the central part of the bulb (Firdaus, 2006). E. palmifolia bulb contains secondary metabolites in the form of naphthoquinone and derivatives such as elecanacine, eleutherine, eleutherol, eleuthernone. Naphthoquinones are known as antimicrobial, antifungal, antiviral, antiparasitic, anticancer, and antioxidants (Hara et al., 1997).

\footnotetext{
*Corresponding author : Roihatul Mutiah

Email : roiha@farmasi.uin-malang.ac.id
}

Other studies have also conducted in Vitro with MTT assay. From this study, it was found that isolates of eleutherinoside $A$ in E. palmifolia bulb which can inhibit $\alpha$-glucosidase in diabetes mellitus (Ieyama, 2011).

This study aims to determine the profile of the metabolites E. palmifolia bulb and determine whether there are differences in the composition of the metabolite compounds E. palmifolia bulb based on different growing conditions. The sampling of $E$. palmifolia bulbs originated from 6 different locations, namely West Java, Central Java, East Java, South Borneo, Central Borneo, and East Borneo. In this study, the metabolite profile of the extract was E. palmifolia bulb analyzed using Ultra Performance Liquid Chromatography - Quadrupole Time of Flight - Mass Spectrum/Mass Spectrometry (UPLC-QToF-MS/MS) which is the best instrument currently for analyzing various compounds. UPLCQToF-MS/MS is an instrument that combines the physical separation capabilities of liquid chromatography with mass analysis capabilities of mass spectrometry. UPLC was chosen because it has the advantage of being well used for many applications that have very high sensitivity and selectivity (Taleuzzaman, 2015). The results of UPLC-QToF-MS/MS will be analyzed using principal component analysis (PCA). PCA is a technique to reduce a lot of data (data grouping) when there are correlations between variables (Rohman, 2009). From the results of PCA visualization, it can be seen that there is a closeness 
Table I. Characteristics of sampling locations of E. palmifolia bulb

\begin{tabular}{|c|c|c|c|c|c|c|}
\hline No. & Location & $\begin{array}{l}\text { Altitude } \\
\text { (MDPL) }\end{array}$ & $\begin{array}{c}\text { Average } \\
\text { Temperature } \\
(\underline{\underline{o}} \mathrm{C}) \\
\end{array}$ & $\begin{array}{l}\text { Rainfall } \\
\text { (mm) }\end{array}$ & Climate & Soil type \\
\hline 1. & $\begin{array}{l}\text { Srengat Village, Srengat } \\
\text { District, Blitar District, East } \\
\text { Java }\end{array}$ & 127 & 25.0 & 1819 & Aw & $\begin{array}{l}\text { Regosol, } \\
\text { Litosol }\end{array}$ \\
\hline 2. & $\begin{array}{l}\text { Kalisoro Village, } \\
\text { Tawangmangu District, } \\
\text { Karanganyar, Central Java }\end{array}$ & 1221 & 19.1 & 3299 & $\mathrm{Am}$ & $\begin{array}{l}\text { Gray Alluvial, } \\
\text { Dark Gray } \\
\text { grumosol }\end{array}$ \\
\hline 3. & $\begin{array}{l}\text { Sukaharja Village, District } \\
\text { Cijeruk, Bogor, West Java }\end{array}$ & 668 & 24.0 & 3454 & Af & $\begin{array}{c}\text { Regosol, } \\
\text { latosol, } \\
\text { Alluvial, } \\
\text { Podsolic, and } \\
\text { Andosol }\end{array}$ \\
\hline 4. & $\begin{array}{l}\text { Karang Rejo, Balikpapan } \\
\text { Tengah Subdistrict, } \\
\text { Balikpapan City, East } \\
\text { Borneo }\end{array}$ & 29 & 26.4 & 2376 & Af & $\begin{array}{l}\text { Red Yellow } \\
\text { Podsolic, and } \\
\text { Sandland }\end{array}$ \\
\hline 5. & $\begin{array}{l}\text { District, Baru Village, Arut } \\
\text { Selatan District, } \\
\text { Kotawaringin Barat } \\
\text { District, Central Borneo }\end{array}$ & 10 & 26.8 & 2765 & Af & $\begin{array}{l}\text { Alluvial, } \\
\text { Latosol, Red } \\
\text { Yellow } \\
\text { Podzolic }\end{array}$ \\
\hline 6. & $\begin{array}{l}\text { District of Banjarbaru, } \\
\text { Banjarmasin, South Borneo }\end{array}$ & 31 & 27.2 & 2627 & Af & Alluvial \\
\hline
\end{tabular}

Source: www.elevationmap.net and https://id.climate-data.org (January 10th, 2018)

between objects (samples) and relationships between variables (Taufik, 2007).

\section{METHODOLOGY \\ Materials}

The plants used in this study were $E$. palmifolia bulb (Eleutherine palmifolia L.) originating from Srengat Village, Srengat District, Blitar Regency, East Java; Kalisoro Village, Tawangmangu District, Karanganyar Regency, Central Java; Sukaharja Village, Cijeruk District, Bogor Regency, West Java; Karang Rejo Village, Balikpapan Tengah District, Balikpapan City, East Borneo; Baru Village, Arut Selatan District, Kotawaringin Barat District, Central Borneo; and Banjarbaru District, Banjarmasin City, South Borneo. The characteristics of the sampling location of E. palmifolia bulb presented in table I.

\section{Determination plants}

Plant determination was carried out at the Materia Medika Office (UPTD) Technical Implementation Unit in Batu, East Java, Indonesia. The number of results of determination of plants is
074/348/102.7/2017. The specimens resulting stored in the Pharmacognosy Laboratory of the Pharmacy Department, Maulana Malik Ibrahim, State Islamic University of Malang.

\section{Instrumentation}

The instruments used were rotary evaporator (IKA, Ohio, USA), ultrasonic cleaner (Sonica Soltec, Milano, Italy), and Ultra Performance Liquid Chromatography-Quadrupole Time of Flight-Mass Spectrometry (UPLC-QToFMS) (Waters, Massachusetts, USA).

\section{Methods}

Extraction

The simplicia of E. palmifolia bulb from each region is 200 grams. Extraction of samples $E$. palmifolia bulb using the maceration method with a combination of Ultrasound-Assisted Extraction (UAE). A sample of 25 grams was dissolved in ethanol $96 \% 500 \mathrm{~mL}$ (comparison 1:20) and replicated three times. The collected filtrate is then separated by its solvent using a rotary evaporator. Next, the extract put in the oven at $40{ }^{\circ} \mathrm{C}$. 
Table II. Gradient Elution System

\begin{tabular}{ccc}
\hline Time (minutes) & \% Eluent A & \% Eluent B \\
\hline 0.00 & 95.0 & 5.0 \\
2.00 & 75.0 & 25.0 \\
3.00 & 75.0 & 25.0 \\
14.00 & 0.0 & 100.0 \\
15.00 & 0.0 & 100.0 \\
19.00 & 95.0 & 5.0 \\
23.00 & 95.0 & 5,0 \\
\hline
\end{tabular}

Table III. Results Extraction Powder E. palmifolia bulbous

\begin{tabular}{lcc}
\hline \multicolumn{1}{c}{ Sample Location } & Extract Weight (g) & Yield (\%) \\
\hline East Java & 8.1034 & 4.0512 \\
Central Java & 10.0483 & 5,0215 \\
West Java & 9.3039 & 4.6517 \\
East Borneo & 12.3269 & 6.1538 \\
Central Borneo & 16.0893 & 8.0430 \\
South Borneo & 10.0772 & 5,0386 \\
\hline
\end{tabular}

Active Compounds Identification using UPLC-QTOF$M S / M S$

The UPLC-QToF-MS/MS analysis used the UPLC-MS system with QToF as an analyzer and positive ESI as an ionization source with columns in the form of Acquity C18, $1.8 \mu \mathrm{m} ; 2.1 \times 150 \mathrm{~mm}$. The eluent used is a mixture of (A) Water (HPLC grade) / formic acid 99.9 / 0.1 [v / v]; (B) Acetonitrile/ formic acid 99.9 / 0.1 [v / v] with a gradient elution system with the following ratio.

The temperature for Source temperature is $100^{\circ} \mathrm{C}$, and desolvation temperature is $350^{\circ} \mathrm{C}$. A total of $10 \mathrm{mg}$ of extract sample was dissolved in a $10 \mathrm{ml}$ volumetric flask with absolute methanol which then injected into the UPLC-MS system, carried out three times repetition. Chromatogram data obtained, and the area expressed in percent form. Chromatograms were processed using software Masslynx version 4.1. Component identification based on measured $\mathrm{m} / \mathrm{z}$ comparisons at Masslynx and $\mathrm{m} / \mathrm{z}$ calculated on ChemDraw.

\section{PCA Multivariate Data Analysis}

Profile data of ethanol extract metabolites 96\% E. palmifolia bulb from UPLC-QToF-MS/MS instruments were then analyzed using PCA. The software used in PCA analysis is Minitab version 17. Data obtained from UPLC-QToF-MS/MS entered into Minitab will then be obtained score plot and loading plot. In the score plot, a graph will appear in the form of small circles. The small circles form groups (groups) specific, and each group indicates the existence of closeness between objects (samples). In the loading plot, a graph will appear in the form of lines of different lengths, some pointing to the left (negative) and the direction to the right (positive). The result of loading the plot used is the line that leads to the right only. The longer the path to the right, the more significant the contribution to differences between groups.

\section{RESULT AND DISCUSSION}

Results of plant extraction

Extraction of E. palmifolia bulb was carried out to obtain a concentrated extract of tuber bulb E. palmifolia. The method used in this research is ultrasound-assisted extraction. This extraction type was chosen because it has several advantages, including faster than cold extraction methods such as maceration and percolation, safer, and can increase the crude yield of extracts (Handayani, 2016).

From Table III, it can be seen that there are differences in the percentage of yield from each region. The difference in returns, other than due to the weight of the extract, can also be influenced by several other factors such as the age of the plant, the time and process of harvesting, the variety of plants, the environment where it grows, and the way of processing plants. These factors can make one of the same plant species finally have a different yield value, the content of the compound is different, and will affect its metabolic activity (Ayunda, 2014; Distantita et al., 2009). 


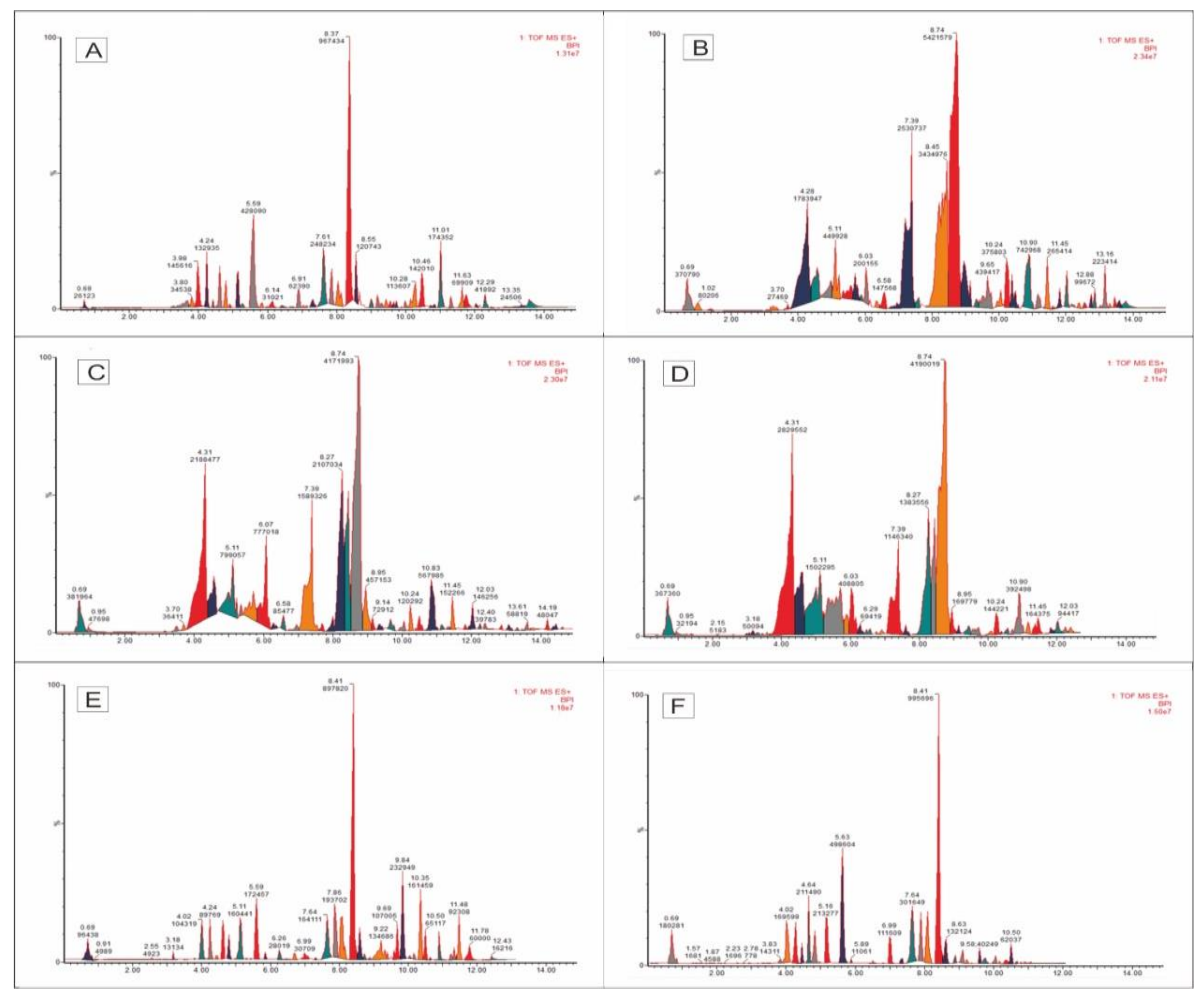

Figure 1. Results of UPLC-QTOF-MS / MS E. palmifolia chromatogram bulb (A: East Java; B: Central Java; C: West Java; D: East Borneo; E: Central Borneo; F: South Borneo).

Active Compounds Identification using UPLC-QTOF$M S / M S$

The content analysis of compounds was $E$. palmifolia bulb carried out using the UPLC-QToFMS/MS type ACQUITY UPLC (instrument Waters). The stationary phase used is C18 or ODS (Octa Desil Silica) which is capable of separating compounds with high, medium, and low polarity levels (Aulia et al, 2016). The mobile phase used is a mixture of formic acid with water $0.1 / 99.9(\mathrm{v} / \mathrm{v})$ and formic acid with acetonitrile 0.1/99.9 (v/v) with a gradient elution system, i.e. the ratio of the two solvents changes change every time. The elution results in the next stage will go to the MS detector. The sample that enters the MS system will turn into a grain of water that drips through the needle and will be added by a positive charge because the ion source used is ESI positive. The results of the separation will appear in the form of a chromatogram which can then be processed using the application Masslynk version 4.1so that the $\mathrm{m} / \mathrm{z}$ spectra can be obtained from each chromatogram peak (Annisa, 2012). The results of the chromatogram of the extract are E. palmifolia bulb shown in Figure 1.

Each peak of the chromatogram indicates the presence of one compound. Chromatograms were processed using the application Masslynx version 4.1 so that the $\mathrm{m} / \mathrm{z}$ spectra could be identified, thus predicting the molecular formula of the result of the interpretation. The prediction of the molecular formula then searched for the name of the compound with the help of the website ChemSpider. When writing the molecular formula to the website of the ChemSpider, the number of molecules $\mathrm{H}$ minus 1 . This is because the source of the ESI ion positive will add the charge $\mathrm{H}$ to the compound in question, so the number of $\mathrm{m} / \mathrm{z}$ must also be reduced by the actual mass of $\mathrm{H}$ at 1,0078 . After obtaining the name of the compound and its structure through the website, compared the measured (measured) $\mathrm{m} / \mathrm{z}$ with calculated (calculated) $\mathrm{m} / \mathrm{z}$ by drawing the structure of the compound in question in the application ChemDraw Ultra 12.0 (Skoog, 2004). If the difference between the two $\leq 0,0005$ then it can be stated that the peak belongs to the predicted compound (Brenton, 2010). The results of the analysis prediction of the active compound on $E$. palmifolia bulb can be seen in Table IV.

The metabolite profile obtained from the results of interpretation (table IV) is that there are differences in the number and part of the compounds. With details of 40 compounds from the East Java sample, 32 compounds from the Central Java sample, 39 compounds from the West 
Table IVa. Prediction of compounds from the interpretation of the data of the chromatogram in each region sampling.

\begin{tabular}{|c|c|c|c|c|c|}
\hline \multicolumn{6}{|c|}{ E. PALMIFOLIA BULB OF EAST JAVA } \\
\hline Rt & \%Area & $\begin{array}{c}\text { Measured } \\
\text { Mass }\end{array}$ & $\begin{array}{l}\text { Calculated } \\
\text { Mass }\end{array}$ & $\begin{array}{l}\text { Molecular } \\
\text { Formula }\end{array}$ & IUPAC Name \\
\hline 0.689 & $0,7001 \%$ & 103,0996 & 103,0997 & $\mathrm{C}_{5} \mathrm{H}_{13} \mathrm{NO}$ & (2S)-2-Amino-3-methyl-1-butanol \\
\hline 0.952 & $0,2750 \%$ & 269,1490 & 269,1488 & $\mathrm{C}_{11} \mathrm{H}_{19} \mathrm{~N}_{5} \mathrm{O}_{3}$ & $\begin{array}{c}2-\{[4,6 \text {-Bis(ethylamino)-1,3,5-triazin-2- } \\
\text { yl]oxy\}ethyl acetate }\end{array}$ \\
\hline 1.352 & $0,3901 \%$ & 120,0323 & 120,0324 & $\mathrm{C}_{6} \mathrm{H}_{4} \mathrm{~N}_{2} \mathrm{O}$ & 2-hydroxynicotinonitrile \\
\hline 2.415 & $0,1434 \%$ & 120,0324 & 120,0324 & $\mathrm{C}_{6} \mathrm{H}_{4} \mathrm{~N}_{2} \mathrm{O}$ & 2-hydroxynicotinonitrile \\
\hline 2.781 & $0,0141 \%$ & 120,0324 & 120,0324 & $\mathrm{C}_{6} \mathrm{H}_{4} \mathrm{~N}_{2} \mathrm{O}$ & 2-hydroxynicotinonitrile \\
\hline 3.284 & $0,1293 \%$ & 149,1203 & 149,1205 & $\mathrm{C}_{10} \mathrm{H}_{15} \mathrm{~N}$ & $\begin{array}{c}\text { 4-(2-Methyl-2-propanyl)aniline } \\
\text { 2-(\{4-Benzyl-5-[1- }\end{array}$ \\
\hline 3.650 & $1,6126 \%$ & 431,2724 & 431,2819 & $\mathrm{C}_{23} \mathrm{H}_{37} \mathrm{~N}_{5} \mathrm{OS}$ & $\begin{array}{l}\text { (dimethylamino)ethyl]-4H-1,2,4-triazol- } \\
\text { 3-yl\}sulfanyl)-N,N-diisobutylacetamide }\end{array}$ \\
\hline 3.798 & $0,9256 \%$ & 475,3003 & 475,2998 & $\begin{array}{l}\mathrm{C}_{16} \mathrm{H}_{42} \mathrm{~N}_{9} \mathrm{O}_{5} \\
\mathrm{Cl}\end{array}$ & UNKNOWN \\
\hline 3.981 & $3,9026 \%$ & 418,1279 & 418,1277 & $\mathrm{C}_{22} \mathrm{H}_{18} \mathrm{~N}_{4} \mathrm{O}_{5}$ & $\begin{array}{c}\text { 2-Methyl-N- }(4-\{[2-(3- \\
\text { nitrobenzoyl)hydrazino]carbonyl }\} \text { pheny } \\
\text { l)benzamide }\end{array}$ \\
\hline 4.244 & $3,5627 \%$ & 585,2064 & 585,2060 & $\mathrm{C}_{32} \mathrm{H}_{27} \mathrm{~N}_{9} \mathrm{OS}$ & $\begin{array}{c}\text { UNKNOWN } \\
\text { L-Phenvlalanvl-J-leucyl-L-servl-N-[(2S)- }\end{array}$ \\
\hline 4.427 & $0,4200 \%$ & 578,3432 & 578,3428 & $\mathrm{C}_{28} \mathrm{H}_{46} \mathrm{~N}_{6} \mathrm{O}_{7}$ & $\begin{array}{c}\text { 6-amino-1-oxo-2-hexanyl]-L- } \\
\text { threoninamide }\end{array}$ \\
\hline 4.610 & $2,9136 \%$ & 636,2038 & 636,2043 & $\mathrm{C}_{35} \mathrm{H}_{32} \mathrm{~N}_{4} \mathrm{O}_{6} \mathrm{~S}$ & $\begin{array}{c}\text { 4-[Bis(5-hydroxy-3-methyl-1-phenyl- } \\
\text { 1H-pyrazol-4-yl)methyl]-2- } \\
\text { methoxyphenyl 4- } \\
\text { methylbenzenesulfonate }\end{array}$ \\
\hline 4.793 & $1,7474 \%$ & 615,2515 & 615,2516 & $\mathrm{C}_{33} \mathrm{H}_{37} \mathrm{~N}_{5} \mathrm{O}_{5} \mathrm{~S}$ & $\begin{array}{c}\mathrm{N}-\{[5-\{[2-(2,3-\text { Dihydro-1H-indol-1-yl)- } \\
\text { 2-oxoethyl]sulfanyl }\}-4-(3- \\
\text { methylphenyl)-4H-1,2,4-triazol-3- } \\
\text { yl]methyl\}-3,4,5-triethoxybenzamide }\end{array}$ \\
\hline 4.896 & $0,2605 \%$ & 244,0735 & 244,0736 & $\mathrm{C}_{14} \mathrm{H}_{12} \mathrm{O}_{4}$ & 1,4-Naphthalenediyl diacetate \\
\hline 5.159 & $3,7501 \%$ & 615,2534 & 615,2532 & $\begin{array}{l}\mathrm{C}_{13} \mathrm{H}_{37} \mathrm{~N}_{13} \mathrm{O}_{1} \\
5\end{array}$ & UNKNOWN \\
\hline 5.262 & $0,3457 \%$ & 383,1345 & 383,1342 & $\mathrm{C}_{17} \mathrm{H}_{17} \mathrm{~N}_{7} \mathrm{O}_{4}$ & $\begin{array}{c}\text { 7-[(2E)-2-Buten-1-yl]-3-methyl-8-[(2E)- } \\
\text { 2-(3-nitrobenzylidene)hydrazino]-3,7- } \\
\text { dihydro-1H-purine-2,6-dione }\end{array}$ \\
\hline 5.594 & $\begin{array}{c}11,4730 \\
\%\end{array}$ & 256,0742 & 256,0743 & $\mathrm{C}_{8} \mathrm{H}_{12} \mathrm{~N}_{6} \mathrm{O}_{2} \mathrm{~S}$ & $\begin{array}{l}4-\left(\mathrm{N}^{\prime}-\right. \\
\text { Carbamimidoylcarbamimidamido)benze } \\
\text { nesulfonamide }\end{array}$ \\
\hline 5.845 & $0,4623 \%$ & 272,1057 & 272,1062 & $\mathrm{C}_{17} \mathrm{H}_{12} \mathrm{~N}_{4}$ & $\begin{array}{l}\text { 2,6-Diphenylimidazo[1,2- } \\
\text { b][1,2,4]triazine } \\
\text { Methyl 4-ethyl-5-methyl-2-(\{[4- }\end{array}$ \\
\hline 6.142 & $0,8314 \%$ & 425,1441 & 425,1443 & $\begin{array}{l}\text { C19H27N3 } \\
\text { O4S2 }\end{array}$ & $\begin{array}{l}\text { Methyl 4-ethyl-5-methyl-2- }(\{[4- \\
\text { (tetrahydro-2-furanylcarbonyl)-1- } \\
\text { piperazinyl]carbonothioyl\}amino)-3- } \\
\text { thiophenecarboxylate }\end{array}$ \\
\hline 6.394 & 0,2388\% & 244,1311 & 244,1311 & C12H2005 & $\begin{array}{c}\text { 4-(3-Methoxy-3-oxopropyl)-2,2- } \\
\text { dimethyltetrahydro-2H-pyran-4- } \\
\text { carboxylic acid }\end{array}$ \\
\hline 6.725 & $0,1201 \%$ & 541,1953 & 541,1948 & $\begin{array}{l}\mathrm{C} 28 \mathrm{H} 31 \mathrm{NO} \\
10\end{array}$ & $\begin{array}{l}\text { (2E)-2,3-Dihydroxy-2-butenedioic acid - } \\
\text { ethyl 5-hydroxy-4-[(3-methyl-1- } \\
\text { piperidinyl)methyl]-2-phenyl-1- } \\
\text { benzofuran-3-carboxylate }\end{array}$ \\
\hline
\end{tabular}


Table IVb. Prediction of compounds from the interpretation of the data of the chromatogram in each region sampling.

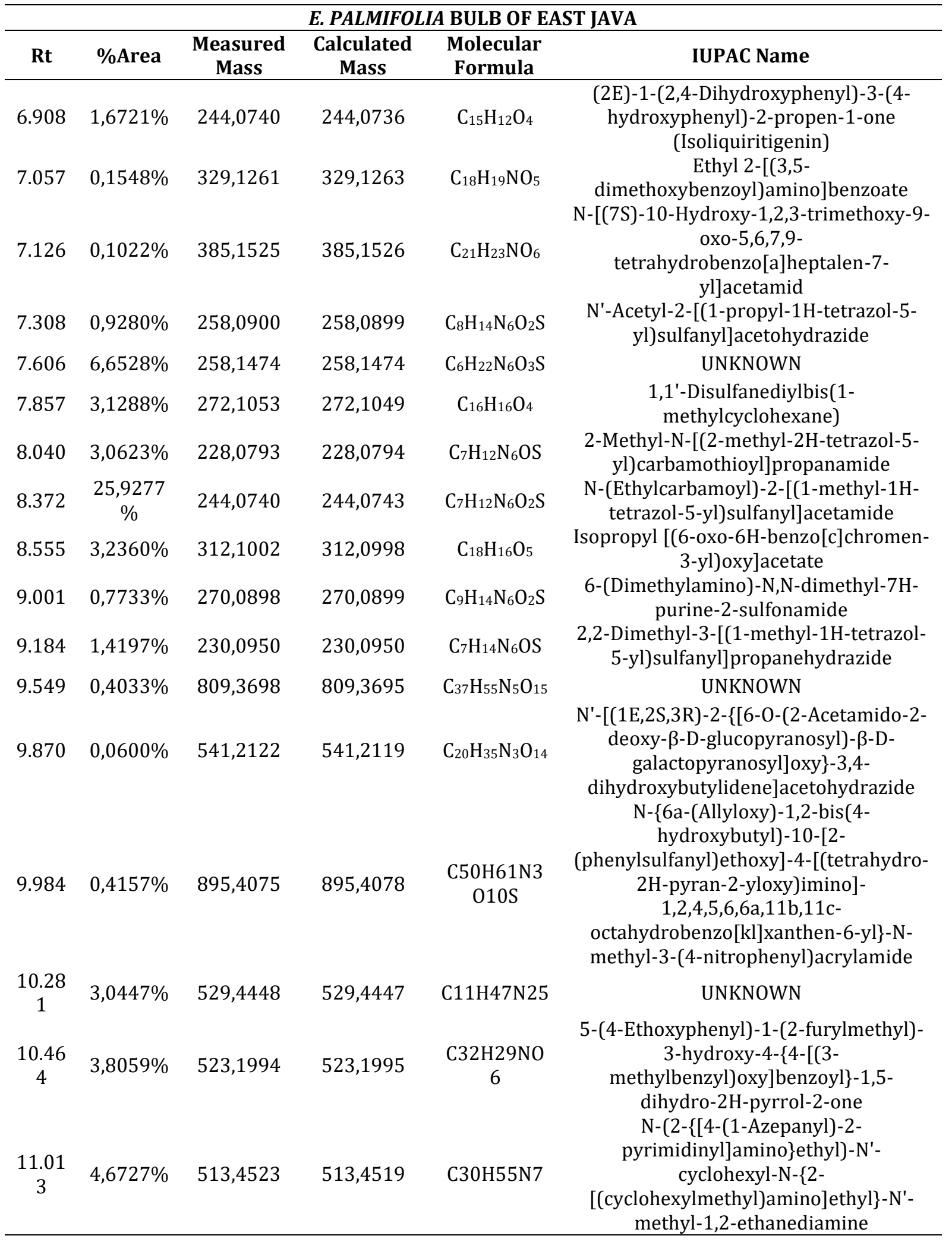


Table IVc. Prediction of compounds from the interpretation of the data of the chromatogram in each region sampling.

\begin{tabular}{|c|c|c|c|c|c|}
\hline \multicolumn{6}{|c|}{ E. PALMIFOLIA BULB OF EAST JAVA } \\
\hline Rt & \%Area & $\begin{array}{c}\text { Measured } \\
\text { Mass }\end{array}$ & $\begin{array}{c}\text { Calculated } \\
\text { Mass }\end{array}$ & $\begin{array}{l}\text { Molecular } \\
\text { Formula }\end{array}$ & IUPAC Name \\
\hline $\begin{array}{c}11.29 \\
9\end{array}$ & $0,8062 \%$ & 542,1967 & 542,1968 & $\mathrm{C}_{34} \mathrm{H}_{22} \mathrm{~N}_{8}$ & $\begin{array}{c}\text { 2,2',2",2"'-Benzene-1,2,4,5- } \\
\text { tetrayltetrakis(1H-pyrrolo[2,3- } \\
\text { b]pyridine) }\end{array}$ \\
\hline $\begin{array}{c}11.74 \\
5\end{array}$ & $1,5604 \%$ & 300,1342 & 300,1340 & $\mathrm{C}_{12} \mathrm{H}_{25} \mathrm{O}_{6} \mathrm{Cl}$ & $\begin{array}{c}\text { 17-Chloro-3,6,9,12,15- } \\
\text { pentaoxaheptadecan-1-ol }\end{array}$ \\
\hline $\begin{array}{c}12.03 \\
0\end{array}$ & $0,2794 \%$ & 541,4801 & 541,4799 & $\mathrm{C}_{20} \mathrm{H}_{59} \mathrm{~N}_{15} \mathrm{~S}$ & UNKNOWN \\
\hline $\begin{array}{c}12.29 \\
3\end{array}$ & $1,1227 \%$ & 481,4609 & 481,4608 & $\mathrm{C}_{29} \mathrm{H}_{59} \mathrm{~N}_{3} \mathrm{O}_{2}$ & N1,N5-Didodecylglutamamide \\
\hline $\begin{array}{c}12.47 \\
6\end{array}$ & $0,0961 \%$ & 283,3244 & 283,3239 & $\mathrm{C}_{19} \mathrm{H}_{41} \mathrm{~N}$ & N-Methyl-1-octadecanamine \\
\hline $\begin{array}{c}12.72 \\
8\end{array}$ & $0,0145 \%$ & 426,3824 & 426,3822 & $\mathrm{C}_{25} \mathrm{H}_{50} \mathrm{~N}_{2} \mathrm{O}_{3}$ & $\begin{array}{c}\text { \{Dimethyl[3- } \\
\text { (stearoylamino)propyl]ammonio\}acetat } \\
\mathrm{e}\end{array}$ \\
\hline $\begin{array}{c}12.91 \\
1\end{array}$ & $0,2142 \%$ & 555,4345 & 555,4340 & $\mathrm{C}_{18} \mathrm{H}_{53} \mathrm{~N}_{17} \mathrm{OS}$ & UNKNOWN \\
\hline $\begin{array}{c}13.34 \\
5\end{array}$ & $0,6568 \%$ & 254,0945 & 254,0943 & $\mathrm{C}_{16} \mathrm{H}_{14} \mathrm{O}_{3}$ & $\begin{array}{l}\text { (2E)-1-(2-Hydroxyphenyl)-3-(4- } \\
\text { methoxyphenyl)prop-2-en-1-one }\end{array}$ \\
\hline $\begin{array}{c}13.64 \\
2\end{array}$ & $1,5715 \%$ & 254,0950 & 254,0950 & $\mathrm{C}_{9} \mathrm{H}_{14} \mathrm{~N}_{6} \mathrm{OS}$ & $\begin{array}{l}\mathrm{N}-(1 \mathrm{H}-\mathrm{Tetrazol}-5- \\
\text { ylcarbamothioyl)cyclohexanecarboxami } \\
\text { de }\end{array}$ \\
\hline $\begin{array}{c}11.29 \\
9\end{array}$ & $0,8062 \%$ & 542,1967 & 542,1968 & $\mathrm{C}_{34} \mathrm{H}_{22} \mathrm{~N}_{8}$ & $\begin{array}{c}\text { 2,2',2",2"'-Benzene-1,2,4,5- } \\
\text { tetrayltetrakis(1H-pyrrolo[2,3- } \\
\text { b]pyridine) }\end{array}$ \\
\hline $\begin{array}{c}11.74 \\
5\end{array}$ & $1,5604 \%$ & 300,1342 & 300,1340 & $\mathrm{C}_{12} \mathrm{H}_{25} \mathrm{O}_{6} \mathrm{Cl}$ & $\begin{array}{c}\text { 17-Chloro-3,6,9,12,15- } \\
\text { pentaoxaheptadecan-1-ol }\end{array}$ \\
\hline $\begin{array}{c}12.03 \\
0\end{array}$ & $0,2794 \%$ & 541,4801 & 541,4799 & $\mathrm{C}_{20} \mathrm{H}_{59} \mathrm{~N}_{15} \mathrm{~S}$ & UNKNOWN \\
\hline $\begin{array}{c}12.29 \\
3\end{array}$ & $1,1227 \%$ & 481,4609 & 481,4608 & $\mathrm{C}_{29} \mathrm{H}_{59} \mathrm{~N}_{3} \mathrm{O}_{2}$ & N1,N5-Didodecylglutamamide \\
\hline $\begin{array}{c}12.47 \\
6\end{array}$ & $0,0961 \%$ & 283,3244 & 283,3239 & $\mathrm{C}_{19} \mathrm{H}_{41} \mathrm{~N}$ & N-Methyl-1-octadecanamine \\
\hline $\begin{array}{c}12.72 \\
8\end{array}$ & $0,0145 \%$ & 426,3824 & 426,3822 & $\mathrm{C}_{25} \mathrm{H}_{50} \mathrm{~N}_{2} \mathrm{O}_{3}$ & $\begin{array}{c}\text { \{Dimethyl[3- } \\
\text { (stearoylamino)propyl]ammonio\}acetat } \\
\mathrm{e}\end{array}$ \\
\hline $\begin{array}{c}12.91 \\
1\end{array}$ & $0,2142 \%$ & 555,4345 & 555,4340 & $\mathrm{C}_{18} \mathrm{H}_{53} \mathrm{~N}_{17} \mathrm{OS}$ & UNKNOWN \\
\hline $\begin{array}{c}13.34 \\
5\end{array}$ & $0,6568 \%$ & 254,0945 & 254,0943 & $\mathrm{C}_{16} \mathrm{H}_{14} \mathrm{O}_{3}$ & $\begin{array}{l}\text { (2E)-1-(2-Hydroxyphenyl)-3-(4- } \\
\text { methoxyphenyl)prop-2-en-1-one }\end{array}$ \\
\hline \multicolumn{6}{|c|}{ E. PALMIFOLIA BULB OF WEST JAVA } \\
\hline Rt & \%Area & $\begin{array}{c}\text { Measured } \\
\text { Mass }\end{array}$ & $\begin{array}{c}\text { Calculated } \\
\text { Mass }\end{array}$ & $\begin{array}{l}\text { Molecular } \\
\text { Formula }\end{array}$ & Nama IUPAC \\
\hline 0.689 & $2,2557 \%$ & 380,0729 & 380,0727 & $\mathrm{C}_{22} \mathrm{H}_{20} \mathrm{~S}_{3}$ & $\begin{array}{l}\text { 4,5-Bis(phenylsulfanyl)-2,3,4,5- } \\
\text { tetrahydro-1-benzothiepine } \\
\text { N-(1H-Benzotriazol-1- }\end{array}$ \\
\hline 0.952 & $0,2817 \%$ & 267,0976 & 267,0973 & $\mathrm{C}_{11} \mathrm{H}_{9} \mathrm{~N}_{9}$ & $\begin{array}{c}\text { ylmethyl)tetrazolo[1,5-b]pyridazin-6- } \\
\text { amine }\end{array}$ \\
\hline
\end{tabular}




\begin{tabular}{|c|c|c|c|c|c|}
\hline \multicolumn{6}{|c|}{ E. PALMIFOLIA BULB OF WEST JAVA } \\
\hline Rt & $\%$ Area & $\begin{array}{c}\text { Measured } \\
\text { Mass }\end{array}$ & $\begin{array}{c}\text { Calculated } \\
\text { Mass }\end{array}$ & $\begin{array}{l}\text { Molecular } \\
\text { Formula }\end{array}$ & Nama IUPAC \\
\hline 1.386 & $0,0129 \%$ & 327,1309 & 327,1305 & $\begin{array}{l}\text { C12H13N11 } \\
0\end{array}$ & UNKNOWN \\
\hline 1.935 & $0,0071 \%$ & 181,9778 & 181,9778 & $\mathrm{CH} 3 \mathrm{~N} 6 \mathrm{OSCl}$ & UNKNOWN \\
\hline 2.118 & $0,0057 \%$ & 181,9779 & 181,9778 & $\mathrm{CH} 3 \mathrm{~N} 6 \mathrm{OSCl}$ & UNKNOWN \\
\hline 2.815 & $0,0026 \%$ & 292,0584 & 292,0583 & C14H12O7 & $\begin{array}{c}\text { [7-(Carboxymethoxy)-4-methyl-2-oxo- } \\
\text { 2H-chromen-3-yl]acetic acid }\end{array}$ \\
\hline 3.147 & $0,0325 \%$ & 396,142 & 396,1421 & С19H2409 & $\begin{array}{l}\text { Methyl 2,3-di-0-acetyl-4,6-0-(4- } \\
\text { methoxybenzylidene)- } \alpha \text {-D- } \\
\text { glucopyranoside }\end{array}$ \\
\hline 3.513 & $0,2311 \%$ & 438,1159 & 438,1162 & $\mathrm{C} 20 \mathrm{H} 22 \mathrm{O} 11$ & $\begin{array}{c}\text { 4-( } \beta \text {-D-Glucopyranosyloxy)-3- } \\
\text { hydroxybenzyl 3,4-dihydroxybenzoate }\end{array}$ \\
\hline 3.696 & $0,2150 \%$ & 292,0591 & 292,0592 & $\begin{array}{l}\mathrm{C} 15 \mathrm{H} 16 \mathrm{O} 2 \mathrm{~S} \\
2\end{array}$ & $\begin{array}{l}\text { 1,1'-(2,2-Propanediyldi-5,2- } \\
\text { thienediyl)diethanone }\end{array}$ \\
\hline 4.313 & $\begin{array}{c}12,9240 \\
\%\end{array}$ & 418,1268 & 418,1264 & С21H22O9 & $\begin{array}{l}\text { 8,8'-(2-Furylmethylene) bis }(6,10- \\
\text { dioxaspiro[4.5]decane-7,9-dione) }\end{array}$ \\
\hline 4.565 & $2,9606 \%$ & 244,0739 & 244,0743 & $\begin{array}{l}\text { C7H12N60 } \\
\text { 2S }\end{array}$ & $\begin{array}{l}\mathrm{N} \text {-(Ethylcarbamoyl)-2-[(1-methyl-1H- } \\
\text { tetrazol-5-yl)sulfanyl]acetamide }\end{array}$ \\
\hline 5.113 & $4,7188 \%$ & 596,2099 & 596,2101 & $\begin{array}{l}\text { C41H28N2 } \\
\quad 03\end{array}$ & $\begin{array}{c}\text { 1,3-Bis(2'-hydroxy-1,1'-binaphthalen-2- } \\
\text { yl)urea }\end{array}$ \\
\hline 5.342 & $0,2960 \%$ & 214,0998 & 214,0994 & C14H1402 & 4,4'-Dimethoxybiphenyl \\
\hline 5.708 & $2,4517 \%$ & 256,1104 & 256,1100 & C16H16O3 & $\begin{array}{l}\text { 4-(Benzyloxy)-3-ethoxybenzaldehyde } \\
\text { (2E)-1-(2,4-Dihydroxyphenyl)-3-(4- }\end{array}$ \\
\hline 6.074 & $4,5887 \%$ & 256,0739 & 256,0736 & C15H12O4 & $\begin{array}{l}\text { hydroxyphenyl)-2-propen-1-one } \\
\text { (Isoliquiritigenin) }\end{array}$ \\
\hline 6.291 & $0,2831 \%$ & 272,1051 & 272,1049 & C16H16O4 & $\begin{array}{c}\text { 2-Hydroxy-1,2-bis(4- } \\
\text { methoxyphenyl)ethanone }\end{array}$ \\
\hline 6.577 & $0,5048 \%$ & 287,1155 & 287,1158 & $\begin{array}{l}\text { C16H17NO } \\
4\end{array}$ & $\begin{array}{l}\text { Methyl 5-(1-acetamido-2-phenylethyl)- } \\
\text { 2-furoate }\end{array}$ \\
\hline 6.943 & $0,3376 \%$ & 244,0737 & 244,0736 & C14H12O4 & $\begin{array}{c}\text { 4-[(E)-2-(3,5-Dihydroxyphenyl)vinyl]- } \\
\text { 1,3-benzenediol } \\
\text { (Oxyresveratrol) }\end{array}$ \\
\hline 7.389 & $9,3857 \%$ & 244,0736 & 244,0740 & $\begin{array}{c}\text { CH6N100Cl } \\
2\end{array}$ & UNKNOWN \\
\hline 7.674 & $0,3336 \%$ & 258,0899 & 258,0899 & $\begin{array}{l}\mathrm{C} 9 \mathrm{H} 20 \mathrm{~N} 2 \mathrm{O} \\
\quad 2 \mathrm{Cl} 2\end{array}$ & $\begin{array}{l}\text { N'-Acetyl-2-[(1-propyl-1H-tetrazol-5- } \\
\text { yl)sulfanyl]acetohydrazide }\end{array}$ \\
\hline 8.269 & $\begin{array}{c}12,4431 \\
\%\end{array}$ & 228,0788 & 228,0787 & C14H12O3 & $\begin{array}{c}\text { 5-[(E)-2-(4-Hydroxyphenyl)vinyl]-1,3- } \\
\text { benzenediol } \\
\text { (Trans-resveratrol) }\end{array}$ \\
\hline 8.452 & $8,3760 \%$ & 228,0786 & 228,0787 & C14H12O3 & $\begin{array}{c}5-[(\mathrm{E})-2-(4-\text { Hydroxyphenyl)vinyl]-1,3- } \\
\text { benzenediol } \\
\text { (Trans-resveratrol) }\end{array}$ \\
\hline 8.738 & $\begin{array}{c}24,6376 \\
\%\end{array}$ & 244,0733 & 244,0729 & $\begin{array}{l}\text { C6H16N2O } \\
\quad 6 \mathrm{~S}\end{array}$ & Lysine sulfate (1:1) \\
\hline 8.955 & $2,6997 \%$ & 312,1006 & 312,1011 & $\begin{array}{l}\text { C19H12N4 } \\
\text { O }\end{array}$ & $\begin{array}{c}\text { 8,9-Diphenylfuro[3,2- } \\
\text { e][1,2,4] triazolo[1,5-c]pyrimidine }\end{array}$ \\
\hline 9.138 & $0,4306 \%$ & 363,147 & 363,1471 & $\begin{array}{l}\mathrm{C} 22 \mathrm{H} 21 \mathrm{NO} \\
4\end{array}$ & $\begin{array}{c}\text { Propyl } 4-\{[(2- \\
\text { naphthyloxy)acetyl]amino\}benzoate }\end{array}$ \\
\hline 9.321 & $0,3363 \%$ & 270,0896 & 270,0892 & $\mathrm{C} 16 \mathrm{H} 1404$ & Diphenyl succinate \\
\hline 9.652 & $0,5991 \%$ & 371,1734 & 371,1729 & $\begin{array}{l}\text { CH22N170 } \\
\quad 4 \mathrm{Cl}\end{array}$ & UNKNOWN \\
\hline
\end{tabular}


Metabolite Fingerprinting Eleutherine palmifolia (L.) Merr.

\begin{tabular}{|c|c|c|c|c|c|}
\hline \multicolumn{6}{|c|}{ E. PALMIFOLIA BULB OF WEST JAVA } \\
\hline Rt & \%Area & $\begin{array}{l}\text { Measured } \\
\text { Mass }\end{array}$ & $\begin{array}{l}\text { Calculated } \\
\text { Mass }\end{array}$ & $\begin{array}{l}\text { Molecular } \\
\text { Formula }\end{array}$ & Nama IUPAC \\
\hline $\begin{array}{c}10.05 \\
3\end{array}$ & 0,3888\% & 315,2774 & 315,2774 & $\begin{array}{c}\text { C18H37NO } \\
3\end{array}$ & $\begin{array}{c}\mathrm{N}, \mathrm{N}-\mathrm{Bis}(2- \\
\text { hydroxyethyl)tetradecanamide }\end{array}$ \\
\hline $\begin{array}{c}10.23 \\
5\end{array}$ & $0,7104 \%$ & 529,4463 & 529,4464 & $\begin{array}{c}\mathrm{C} 30 \mathrm{H} 63 \mathrm{~N} 3 \mathrm{~S} \\
2\end{array}$ & UNKNOWN \\
\hline $\begin{array}{c}10.83 \\
0\end{array}$ & $3,3542 \%$ & 356,0903 & 356,0903 & $\begin{array}{l}\text { C12H16N6 } \\
\text { O5S }\end{array}$ & $\begin{array}{c}\text { 5'-Deoxy-5'- } \\
\text { [(vinylsulfonyl)amino]adenosine }\end{array}$ \\
\hline $\begin{array}{c}11.11 \\
6\end{array}$ & $0,2261 \%$ & 258,1261 & 258,1256 & С16Н1803 & $\begin{array}{l}\text { Ethyl 2-(6-methoxy-2- } \\
\text { naphthyl)propanoate }\end{array}$ \\
\hline $\begin{array}{c}11.29 \\
9\end{array}$ & $0,1186 \%$ & 578,286 & 578,2855 & C38H42O3S & UNKNOWN \\
\hline $\begin{array}{c}11.44 \\
7\end{array}$ & $0,8992 \%$ & 497,457 & 497,4575 & C15H51N19 & UNKNOWN \\
\hline $\begin{array}{c}11.81 \\
3\end{array}$ & $0,1298 \%$ & 621,3731 & 621,3729 & $\begin{array}{c}\mathrm{C} 13 \mathrm{H} 39 \mathrm{~N} 27 \\
03\end{array}$ & UNKNOWN \\
\hline $\begin{array}{c}12.03 \\
1\end{array}$ & $0,8637 \%$ & 481,4612 & 481,4608 & $\begin{array}{l}\text { C29H59N3 } \\
\text { O2 }\end{array}$ & N1,N5-Didodecylglutamamide \\
\hline $\begin{array}{c}12.24 \\
8\end{array}$ & $0,1416 \%$ & 533,3566 & 533,3564 & $\begin{array}{l}\text { C27H51NO } \\
9\end{array}$ & $\begin{array}{c}\text { Hexadecyl 5-acetamido-3,5-dideoxy-6- } \\
\text { [(1S,2R)-1,2,3-trihydroxypropyl]hex-2- } \\
\text { ulopyranosidonicacid }\end{array}$ \\
\hline $\begin{array}{c}12.39 \\
6\end{array}$ & $0,2349 \%$ & 416,2319 & 416,2314 & $\begin{array}{l}\text { C16H40N4 } \\
\text { O2S3 }\end{array}$ & UNKNOWN \\
\hline $\begin{array}{c}12.54 \\
5\end{array}$ & $0,0265 \%$ & 408,3751 & 408,3756 & $\mathrm{C} 30 \mathrm{H} 48$ & $\begin{array}{c}\text { (6E,10E,12Z,14E,18E)-2,6,10,15,19,23- } \\
\text { Hexamethyl-2,6,10,12,14,18,22- } \\
\text { tetracosaheptaene }\end{array}$ \\
\hline $\begin{array}{c}12.87 \\
7\end{array}$ & $0,1571 \%$ & 323,282 & 323,2816 & $\begin{array}{c}\text { C15H38N5C } \\
1\end{array}$ & $\begin{array}{l}\text { 4-Amino-N,N-bis(4-aminobutyl)-N-(3- } \\
\text { aminopropyl)-1-butanaminium chloride }\end{array}$ \\
\hline $\begin{array}{c}13.05 \\
9\end{array}$ & $0,2498 \%$ & 392,2324 & 392,2325 & $\begin{array}{c}\mathrm{C} 22 \mathrm{H} 28 \mathrm{~N} 6 \\
\mathrm{O}\end{array}$ & $\begin{array}{l}\mathrm{N}, \mathrm{N} \text {-Diethyl-1-[1-(3-methylphenyl)-1H- } \\
\text { pyrazolo[3,4-d]pyrimidin-4-yl]-3- } \\
\text { piperidinecarboxamide }\end{array}$ \\
\hline $\begin{array}{c}13.31 \\
1\end{array}$ & $0,0830 \%$ & 523,3649 & 523,3645 & $\begin{array}{l}\text { C23H51N9C } \\
12\end{array}$ & UNKNOWN \\
\hline $\begin{array}{c}13.60 \\
8\end{array}$ & $0,3474 \%$ & 278,2249 & 278,2246 & C18H3002 & $\begin{array}{l}(9 \mathrm{E}, 12 \mathrm{E}, 15 \mathrm{E})-9,12,15- \\
\text { Octadecatrienoic acid }\end{array}$ \\
\hline $\begin{array}{c}13.79 \\
1\end{array}$ & $0,1049 \%$ & 412,3701 & 412,3705 & С29H480 & $\begin{array}{c}(3 \alpha, 5 \alpha, 9 \xi, 14 \xi, 22 \mathrm{E}, 24 \mathrm{R})-\text { Stigmasta-7,22- } \\
\text { dien-3-ol }\end{array}$ \\
\hline $\begin{array}{c}14.19 \\
1\end{array}$ & $0,2837 \%$ & 281,2716 & 281,2719 & C18H35NO & 1-Dodecyl-2-azepanone \\
\hline $\begin{array}{c}14.44 \\
3\end{array}$ & $0,2739 \%$ & 470,3401 & 470,3396 & С30H4604 & $\begin{array}{c}(3 \beta, 5 \alpha, 14 \xi, 25 R)-\text { Spirost-9(11)-en-3-yl } \\
\text { propionate }\end{array}$ \\
\hline $\begin{array}{c}14.62 \\
6 \\
\end{array}$ & $0,0545 \%$ & 281,2145 & 281,2144 & $\mathrm{C} 20 \mathrm{H} 27 \mathrm{~N}$ & $\begin{array}{c}\text { (1E)-2,2,5,9-Tetramethyl-N-phenyl- } \\
\text { 3,4,8-decatrien-1-imine }\end{array}$ \\
\hline \multicolumn{6}{|c|}{ E. PALMIFOLIA BULB EAST BORNEO } \\
\hline Rt & \%Area & $\begin{array}{l}\text { Measured } \\
\text { Mass }\end{array}$ & $\begin{array}{c}\text { Calculated } \\
\text { Mass } \\
\end{array}$ & $\begin{array}{c}\text { Molecular } \\
\text { Formula }\end{array}$ & IUPAC Name \\
\hline 0.689 & $2,1443 \%$ & 380,0722 & 380,0719 & $\mathrm{C}_{21} \mathrm{H}_{16} \mathrm{O}_{5} \mathrm{~S}$ & $\begin{array}{l}\text { 4-Methyl-6-oxo-6H-benzo[c]chromen-3- } \\
\text { yl 4-methylbenzenesulfonate }\end{array}$ \\
\hline 0.952 & $0,1879 \%$ & 267,0968 & 267,0968 & $\begin{array}{l}\text { C10H13N5 } \\
\text { O4 }\end{array}$ & Adenosine \\
\hline 1.237 & $0,0270 \%$ & 489,1831 & 489,1830 & $\begin{array}{l}\text { C3H24N25 } \\
\text { Br }\end{array}$ & UNKNOWN \\
\hline
\end{tabular}




\begin{tabular}{|c|c|c|c|c|c|}
\hline \multicolumn{6}{|c|}{ E. PALMIFOLIA BULB EAST BORNEO } \\
\hline Rt & \%Area & $\begin{array}{c}\text { Measured } \\
\text { Mass }\end{array}$ & $\begin{array}{c}\text { Calculated } \\
\text { Mass }\end{array}$ & $\begin{array}{l}\text { Molecular } \\
\text { Formula }\end{array}$ & IUPAC Name \\
\hline 1.386 & $0,0623 \%$ & 165,0783 & 165,0781 & $\mathrm{C} 4 \mathrm{H} 12 \mathrm{~N} 5 \mathrm{Cl}$ & $\begin{array}{l}\text { N,N-Dimethylimidodicarbonimidic } \\
\text { diamide hydrochloride }(1: 1)\end{array}$ \\
\hline 2.152 & $0,0303 \%$ & 187,0633 & 187,0634 & $\begin{array}{l}\mathrm{C} 11 \mathrm{H} 11 \mathrm{NO} \\
2\end{array}$ & 3-(1H-Indol-3-yl)propanoic acid \\
\hline 2.598 & $0,0186 \%$ & 246,0521 & 246,0520 & $\begin{array}{l}\text { C8H11N40 } \\
3 \mathrm{Cl}\end{array}$ & $\begin{array}{l}\text { N-(6-Amino-1,3-dimethyl-2,4-dioxo- } \\
\text { 1,2,3,4-tetrahydro-5-pyrimidinyl)-2- } \\
\text { chloroacetamide }\end{array}$ \\
\hline 3.181 & $0,2924 \%$ & 422,0848 & 422,0849 & С19H18011 & $\begin{array}{c}(1 \xi)-1,5-\text { Anhydro-1-(1,3,6,7- } \\
\text { tetrahydroxy-9-oxo-9H-xanthen-2-yl)- } \\
\text { D-allitol }\end{array}$ \\
\hline 3.284 & $0,0932 \%$ & 438,1159 & 438,1162 & C20H22011 & $\begin{array}{c}\text { 4-( }(\beta \text {-D-Glucopyranosyloxy)-3- } \\
\text { hydroxybenzyl 3,4-dihydroxybenzoate }\end{array}$ \\
\hline 3.433 & $0,0496 \%$ & 396,1415 & 396,1413 & $\begin{array}{l}\text { C19H29N2 } \\
\text { O2Br }\end{array}$ & $\begin{array}{l}\text { 1'-(2-Bromo-4,5-dimethoxybenzyl)-1,4'- } \\
\text { bipiperidine }\end{array}$ \\
\hline 3.581 & $0,0654 \%$ & 418,1256 & 418,1252 & C26H18N4S & $\begin{array}{l}\text { 2-[(1H-Benzimidazol-2- } \\
\text { ylmethyl)sulfanyl]-4,6- } \\
\text { diphenylnicotinonitrile }\end{array}$ \\
\hline 4.313 & $\begin{array}{c}16,5162 \\
\%\end{array}$ & 418,1261 & 418,1264 & C21H2209 & $\begin{array}{l}\text { 8,8'-(2-Furylmethylene) bis }(6,10- \\
\text { dioxaspiro[4.5] decane-7,9-dione })\end{array}$ \\
\hline 4.610 & $6,0092 \%$ & 244,0741 & 244,0736 & C14H1204 & 1,4-Naphthalenediyl diacetate \\
\hline 5.113 & $8,7689 \%$ & 596,2105 & 596,2100 & $\begin{array}{c}\text { C41H28N2 } \\
\text { 03 }\end{array}$ & $\begin{array}{c}\text { 1,3-Bis(2'-hydroxy-1,1'-binaphthalen-2- } \\
\text { yl)urea }\end{array}$ \\
\hline 5.708 & $8,1186 \%$ & 256,1101 & 256,1100 & C16H16O3 & 4-(Benzyloxy)-3-ethoxybenzaldehyde \\
\hline 5.891 & $1,1041 \%$ & 256,0736 & 256,0736 & C15H1204 & $\begin{array}{l}\text { (2E)-1-(2,4-Dihydroxyphenyl)-3-(4- } \\
\text { hydroxyphenyl)-2-propen-1-one } \\
\text { (Isoliquiritigenin) }\end{array}$ \\
\hline 6.028 & $2,3862 \%$ & 256,0744 & 256,0749 & C16H8N4 & $\begin{array}{c}\text { Quinoxalino[2',3':3,4]cyclobuta[1,2- } \\
\text { b]quinoxaline }\end{array}$ \\
\hline 6.291 & $0,4052 \%$ & 272,1051 & 272,1049 & C16H1604 & $\begin{array}{l}\text { 2-Hydroxy-1,2-bis(4- } \\
\text { methoxyphenyl)ethanone }\end{array}$ \\
\hline 6.577 & $0,3111 \%$ & 403,1617 & 403,1618 & $\begin{array}{c}\mathrm{C} 18 \mathrm{H} 17 \mathrm{~N} 11 \\
0\end{array}$ & $\begin{array}{c}\text { [3-(1H-Tetrazol-1-yl)phenyl }]\{4-[6-(1 \mathrm{H}- \\
\text { 1,2,4-triazol-1-yl)-3-pyridazinyl }]-1- \\
\text { piperazinyl }\} \text { methanone }\end{array}$ \\
\hline 6.760 & $0,0546 \%$ & 342,0733 & 342,0732 & $\begin{array}{l}\text { C18H19N2 } \\
\text { BrN2 }\end{array}$ & $\begin{array}{l}\text { Diphenyl }[(3 S)-3- \\
\text { pyrrolidinyl]acetonitrile hydrobromide } \\
(1: 1)\end{array}$ \\
\hline 6.908 & $0,1698 \%$ & 242,0582 & 242,0579 & C14H1004 & $\begin{array}{l}\text { 9,10-Dihydroxy-2,3-dihydro-1,4- } \\
\text { anthracenedione }\end{array}$ \\
\hline 7.389 & $6,6912 \%$ & 244,0738 & 244,0743 & $\begin{array}{l}\text { C7H12N6O } \\
2 \mathrm{~S}\end{array}$ & $\begin{array}{l}\mathrm{N} \text {-(Ethylcarbamoyl)-2-[(1-methyl-1H- } \\
\quad \text { tetrazol-5-yl)sulfanyl]acetamide }\end{array}$ \\
\hline 7.606 & $0,3505 \%$ & 370,2834 & 370,2832 & $\begin{array}{l}\text { C20H38N2 } \\
\text { O4 }\end{array}$ & $\begin{array}{l}\text { Ethyl 4-\{2-hydroxy-3-[(2-isopropyl-5- } \\
\text { methylcyclohexyl)oxy]propyl }\}-1- \\
\text { piperazinecarboxylate }\end{array}$ \\
\hline 8.269 & $8,0759 \%$ & 228,0788 & 228,0787 & C14H12O3 & $\begin{array}{c}5-[(\mathrm{E})-2-(4-\text { Hydroxyphenyl)vinyl }]-1,3- \\
\text { benzenediol } \\
\text { (Trans-resveratrol) }\end{array}$ \\
\hline 8.452 & $5,4174 \%$ & 228,0785 & 228,0787 & C14H1203 & $\begin{array}{c}5-[(\mathrm{E})-2-(4-\text { Hydroxyphenyl)vinyl }]-1,3- \\
\text { benzenediol } \\
\text { (Trans-resveratrol) }\end{array}$ \\
\hline 8.738 & $\begin{array}{c}24,4573 \\
\%\end{array}$ & 244,0739 & 244,0743 & $\begin{array}{l}\text { C7H12N60 } \\
2 \mathrm{~S}\end{array}$ & $\begin{array}{l}\mathrm{N}-(\text { Ethylcarbamoyl)-2-[(1-methyl-1H- } \\
\text { tetrazol-5-yl)sulfanyl]acetamide }\end{array}$ \\
\hline
\end{tabular}


Metabolite Fingerprinting Eleutherine palmifolia (L.) Merr.

\begin{tabular}{|c|c|c|c|c|c|}
\hline \multicolumn{6}{|c|}{ E. PALMIFOLIA BULB EAST BORNEO } \\
\hline $\mathbf{R t}$ & $\%$ Area & $\begin{array}{l}\text { Measured } \\
\text { Mass }\end{array}$ & $\begin{array}{l}\text { Calculated } \\
\text { Mass }\end{array}$ & $\begin{array}{l}\text { Molecular } \\
\text { Formula }\end{array}$ & IUPAC Name \\
\hline 8.955 & $0,9910 \%$ & 312,0999 & 312,0998 & C18H1805 & $\begin{array}{c}\text { (2E)-3-(3,4-Dimethoxyphenyl)-1-(2- } \\
\text { hydroxy-4-methoxyphenyl)-2-propen-1- } \\
\text { one }\end{array}$ \\
\hline 9.435 & $0,2784 \%$ & 363,1466 & 363,1471 & $\begin{array}{c}\mathrm{C} 22 \mathrm{H} 21 \mathrm{NO} \\
4\end{array}$ & $\begin{array}{c}\text { Propyl 4-\{[(2- } \\
\text { naphthyloxy)acetyl]amino\}benzoate }\end{array}$ \\
\hline $\begin{array}{c}10.09 \\
8\end{array}$ & $0,1821 \%$ & 543,4246 & 543,4241 & $\begin{array}{c}\text { C32H62NOS } \\
\mathrm{Cl}\end{array}$ & UNKNOWN \\
\hline $\begin{array}{c}10.23 \\
5\end{array}$ & $0,8418 \%$ & 529,445 & 529,4447 & C11H47N25 & UNKNOWN \\
\hline $\begin{array}{c}10.38 \\
4\end{array}$ & $0,0421 \%$ & 523,1999 & 523,1999 & $\begin{array}{l}\text { C18H45N5S } \\
6\end{array}$ & UNKNOWN \\
\hline $\begin{array}{l}10.56 \\
7\end{array}$ & $0,2405 \%$ & 523,2002 & 523,2002 & $\begin{array}{l}\text { C25H29N7 } \\
\text { O4S }\end{array}$ & $\begin{array}{c}\mathrm{N} \alpha-\{[(2 \mathrm{~S}, 4 \mathrm{~S})-1-[(1-\text { Methyl-1H-pyrrol-2- } \\
\text { yl)carbonyl]-4-\{[(4-methyl-1,2,3- } \\
\text { thiadiazol-5-yl)carbonyl]amino }\}-2- \\
\text { piperidinyl]carbonyl }\}-\mathrm{D}- \\
\text { phenylalaninamide }\end{array}$ \\
\hline $\begin{array}{l}10.89 \\
9\end{array}$ & $2,2910 \%$ & 513,4522 & 513,4519 & C30H55N7 & $\begin{array}{c}\mathrm{N}-(2-\{[4-(1-\text { Azepanyl })-2- \\
\text { pyrimidinyl }] \text { amino }\} \text { ethyl })-\mathrm{N}^{\prime}- \\
\text { cyclohexyl-N- }\{2- \\
\text { [(cyclohexylmethyl)amino]ethyl }\}-\mathrm{N}^{\prime}- \\
\text { methyl-1,2-ethanediamine }\end{array}$ \\
\hline $\begin{array}{c}11.15 \\
0\end{array}$ & $0,4624 \%$ & 258,1257 & 258,1256 & C16H1803 & $\begin{array}{l}\text { Ethyl 2-(6-methoxy-2- } \\
\text { naphthyl)propanoate }\end{array}$ \\
\hline $\begin{array}{c}11.44 \\
7\end{array}$ & $0,9595 \%$ & 497,4556 & 497,4557 & $\begin{array}{l}\text { C29H59N3 } \\
\text { O3 }\end{array}$ & UNKNOWN \\
\hline $\begin{array}{c}11.81 \\
3\end{array}$ & $0,2532 \%$ & 621,3727 & 621,3723 & $\begin{array}{l}\text { C5H43N29 } \\
\text { 05S }\end{array}$ & UNKNOWN \\
\hline $\begin{array}{c}12.03 \\
1\end{array}$ & $0,5511 \%$ & 495,3326 & 495,3322 & $\begin{array}{l}\mathrm{C} 27 \mathrm{H} 41 \mathrm{~N} 7 \\
\mathrm{O} 2\end{array}$ & UNKNOWN \\
\hline $\begin{array}{c}12.39 \\
6\end{array}$ & $0,2398 \%$ & 416,2328 & 533,3567 & $\begin{array}{c}\text { C24H28N6 } \\
0\end{array}$ & $\begin{array}{c}(1 \mathrm{R}, 2 \mathrm{~S})-2-(\{[(2-\text { Methyl-2- } \\
\text { propanyl)oxy] carbonyl }\} \text { amino }) \text { cycloocta } \\
\text { necarboxylic acid - }(1 \mathrm{~S}, 2 \mathrm{R})-2-(\{[(2- \\
\text { methyl-2- } \\
\text { propanyl)oxy] carbonyl }\} \text { amino }) \text { cycloocta } \\
\text { necarboxylic acid }(1: 1) \\
\text { 2-Amino-1-[4-(diethylamino)phenyl]-N- } \\
\text { propyl-1H-pyrrolo[2,3-b]quinoxaline-3- } \\
\text { carboxamide }\end{array}$ \\
\hline \multicolumn{6}{|c|}{ E. PALMIFOLIA BULB CENTRAL BORNEO } \\
\hline Rt & \%Area & $\begin{array}{l}\text { Measured } \\
\text { Mass }\end{array}$ & $\begin{array}{c}\text { Calculated } \\
\text { Mass } \\
\end{array}$ & $\begin{array}{l}\text { Molecular } \\
\text { Formula } \\
\end{array}$ & Nama IUPAC \\
\hline 0.689 & $2,9195 \%$ & 364,0986 & 364,0984 & $\begin{array}{c}\mathrm{C}_{6} \mathrm{H}_{13} \mathrm{~N}_{14} \mathrm{O}_{3} \\
\mathrm{Cl}\end{array}$ & UNKNOWN \\
\hline 2.552 & $0,1490 \%$ & 374,1181 & 374,1186 & $\mathrm{C}_{12} \mathrm{H}_{18} \mathrm{~N}_{6} \mathrm{O}_{8}$ & $\begin{array}{c}\text { 4-[(1E)-3,3-Dimethyl-1-triazen-1-yl]- } \\
\text { 1H-imidazole-5-carboxamide } 2 \text { - } \\
\text { hydroxy-1,2,3-propanetricarboxylate } \\
(1: 1)\end{array}$ \\
\hline 2.781 & $0,0229 \%$ & 124,9784 & 124,9781 & $\mathrm{C} 4 \mathrm{~N} 3 \mathrm{Cl}$ & Chloromethanetricarbonitrile \\
\hline 3.181 & $0,3976 \%$ & 289,1308 & 289,1306 & $\begin{array}{l}\mathrm{C} 11 \mathrm{H} 20 \mathrm{~N} 5 \\
\mathrm{O} 2 \mathrm{Cl}\end{array}$ & $\begin{array}{c}\text { 6-Chloro-N,N'-bis(3-methoxypropyl)- } \\
\text { 1,3,5-triazine-2,4-diamine }\end{array}$ \\
\hline
\end{tabular}




\begin{tabular}{|c|c|c|c|c|c|}
\hline \multicolumn{6}{|c|}{ E. PALMIFOLIA BULB CENTRAL BORNEO } \\
\hline Rt & \%Area & $\begin{array}{l}\text { Measured } \\
\text { Mass }\end{array}$ & $\begin{array}{c}\text { Calculated } \\
\text { Mass }\end{array}$ & $\begin{array}{l}\text { Molecular } \\
\text { Formula }\end{array}$ & Nama IUPAC \\
\hline 3.284 & $0,0266 \%$ & 289,1308 & 289,1306 & $\begin{array}{l}\text { C11H20N5 } \\
\text { O2Cl }\end{array}$ & $\begin{array}{c}\text { 6-Chloro-N,N'-bis(3-methoxypropyl)- } \\
\text { 1,3,5-triazine-2,4-diamine }\end{array}$ \\
\hline 3.433 & $0,0279 \%$ & 124,9781 & 124,9781 & $\mathrm{C} 4 \mathrm{~N} 3 \mathrm{Cl}$ & Nitromethanesulfinic acid \\
\hline 3.650 & $0,0382 \%$ & 124,978 & 124,9783 & CH3NO4S & Nitromethanesulfinic acid \\
\hline 3.799 & $0,0742 \%$ & 475,2994 & 475,2991 & $\begin{array}{l}\text { C19H47N7S } \\
\text { Cl2 }\end{array}$ & UNKNOWN \\
\hline 4.016 & $3,1581 \%$ & 418,1263 & 418,1264 & $\mathrm{C} 21 \mathrm{H} 2209$ & $\begin{array}{l}\text { 8,8'-(2-Furylmethylene)bis(6,10- } \\
\text { dioxaspiro[4.5]decane-7,9-dione) }\end{array}$ \\
\hline 4.244 & $2,7176 \%$ & 590,1608 & 590,1609 & $\begin{array}{l}\text { C24H26N6 } \\
012\end{array}$ & $\begin{array}{c}\text { Diisopropyl 4,4'-(1,4- } \\
\text { piperazinediyl)bis(3,5-dinitrobenzoate) } \\
\text { Methyl }(2 \mathrm{R})-2-\{(1 \mathrm{~S}, 2 \mathrm{E}, 3 \mathrm{~S}, 4 \mathrm{R})-2-\end{array}$ \\
\hline 4.462 & $0,3536 \%$ & 372,1792 & 372,1793 & $\begin{array}{l}\text { C19H32O3S } \\
2\end{array}$ & $\begin{array}{c}\text { (methoxymethylene)-4-methyl-3-[2-(2- } \\
\text { methyl-1,3-dithiolan-2- } \\
\text { yl)ethyl]cyclohexyl\}propanoate }\end{array}$ \\
\hline 4.610 & $3,0357 \%$ & 636,2026 & 636,2028 & $\begin{array}{l}\text { C26H32N6 } \\
013\end{array}$ & $\begin{array}{c}\text { (2R)-4-\{4,6-Dimethyl-9-oxo-3-[(2,3,5- } \\
\text { tri-0-acetyl- } \beta \text {-D-ribofuranosyl)oxy]-4,9- } \\
\text { dihydro-3H-imidazo[1,2-a]purin-7-yl\}- } \\
\text { 2-[(methoxycarbonyl)amino]butanoic } \\
\text { acid }\end{array}$ \\
\hline 4.793 & $1,4541 \%$ & 620,2087 & 620,2092 & $\begin{array}{l}\text { C27H28N10 } \\
\text { O8 }\end{array}$ & $\begin{array}{c}\text { 4-[(E)-(\{[1-(4-Amino-1,2,5-oxadiazol-3- } \\
\text { yl)-4-(4-morpholinylmethyl)-1H-1,2,3- } \\
\text { triazol-5- } \\
\text { yl]carbonyl\}hydrazono)methyl]-2- } \\
\text { ethoxyphenyl 3-methyl-4-nitrobenzoate }\end{array}$ \\
\hline 5.113 & $4,8572 \%$ & 620,2078 & 620,2078 & $\begin{array}{l}\text { C26H32N6 } \\
012\end{array}$ & $\begin{array}{l}\text { Methyl 2-azido-4,6-0-benzylidene-2- } \\
\text { deoxy-3-0-(3,4,6-tri-O-acetyl-2-azido-2- } \\
\text { deoxy- } \alpha \text {-D-galactopyranosyl)- } \beta \text {-D- } \\
\text { galactopyranoside }\end{array}$ \\
\hline 5.296 & $0,0673 \%$ & 321,1352 & 321,1350 & $\begin{array}{l}\text { C7H24N7O } \\
\quad 3 S C l\end{array}$ & UNKNOWN \\
\hline 5.445 & $0,0150 \%$ & 124,9782 & 124,9781 & $\mathrm{C} 4 \mathrm{~N} 3 \mathrm{Cl}$ & $\begin{array}{l}\text { Chloromethanetricarbonitrile } \\
\text { (2E)-1-(2,4-Dihydroxyphenyl)-3-(4- }\end{array}$ \\
\hline 5.594 & $5,2209 \%$ & 256,0735 & 256,0736 & C15H12O4 & $\begin{array}{l}\text { hydroxyphenyl)-2-propen-1-one } \\
\text { (Isoliquiritigenin) }\end{array}$ \\
\hline 5.845 & 0,4397\% & 272,1044 & 272,1049 & C16H16O4 & $\begin{array}{l}\text { 2-Hydroxy-1,2-bis }(4- \\
\text { methoxyphenyl)ethanone }\end{array}$ \\
\hline 6.257 & $0,8482 \%$ & 279,0894 & 279,0896 & $\begin{array}{c}\text { C17H13NO } \\
3\end{array}$ & $\begin{array}{l}\text { 2-(1-Oxo-1-phenyl-2-propanyl)-1H- } \\
\text { isoindole-1,3(2H)-dione }\end{array}$ \\
\hline 6.474 & $0,1076 \%$ & 242,0573 & 242,0573 & $\begin{array}{l}\text { C6H14N2O } \\
\quad 6 \mathrm{~S}\end{array}$ & $\begin{array}{l}\text { 6-Deoxy-N-sulfamoyl- } \beta \text {-D- } \\
\text { mannopyranosylamine }\end{array}$ \\
\hline 6.691 & $0,5581 \%$ & 259,0835 & 259,0836 & $\begin{array}{l}\text { C9H14N5O } \\
2 \mathrm{Cl}\end{array}$ & $\begin{array}{l}\text { 2-\{[4-Chloro-6-(4-morpholinyl)-1,3,5- } \\
\text { triazin-2-yl]amino\}ethanol } \\
\text { 4-[(E)-2-(3,5-Dihydroxyphenyl)vinyl]- }\end{array}$ \\
\hline 6.988 & $0,9297 \%$ & 244,0734 & 244,0736 & C14H12O4 & $\begin{array}{l}\text { 1,3-benzenediol } \\
\text { (Oxyresveratrol) }\end{array}$ \\
\hline 7.308 & $0,0982 \%$ & 258,089 & 258,0892 & C15H14O4 & $\begin{array}{l}\text { (2E)-1-(2,4-Dihydroxyphenyl)-3-(4- } \\
\text { hydroxyphenyl)-2-propen-1-one } \\
\text { (Isoliquiritigenin) }\end{array}$ \\
\hline 7.640 & $4,9683 \%$ & 258,1467 & 258,1467 & C13H22O5 & $\begin{array}{l}\text { tert-Butyl 3,5-dideoxy-2,4-0- } \\
\text { isopropylidene-L-erythro-hexuronate }\end{array}$ \\
\hline 7.857 & $5,8641 \%$ & 272,1049 & 272,1049 & C16H16O4 & $\begin{array}{l}\text { 2-Hydroxy-1,2-bis(4- } \\
\text { methoxyphenyl)ethanone }\end{array}$ \\
\hline
\end{tabular}


Metabolite Fingerprinting Eleutherine palmifolia (L.) Merr.

\begin{tabular}{|c|c|c|c|c|c|}
\hline \multicolumn{6}{|c|}{ E. PALMIFOLIA BULB CENTRAL BORNEO } \\
\hline Rt & \%Area & $\begin{array}{c}\text { Measured } \\
\text { Mass }\end{array}$ & $\begin{array}{c}\text { Calculated } \\
\text { Mass }\end{array}$ & $\begin{array}{l}\text { Molecular } \\
\text { Formula }\end{array}$ & Nama IUPAC \\
\hline 8.086 & $5,6093 \%$ & 228,0784 & 228,0787 & C14H12O3 & $\begin{array}{l}\text { 5-[(E)-2-(4-Hydroxyphenyl)vinyl]-1,3- } \\
\text { benzenediol }\end{array}$ \\
\hline 8.406 & $\begin{array}{c}27,1804 \\
\%\end{array}$ & 244,0733 & 244,0729 & $\begin{array}{l}\text { C6H16N20 } \\
\quad 6 \mathrm{~S}\end{array}$ & Lysine sulfate $(1: 1)$ \\
\hline 8.589 & $3,1634 \%$ & 312,0986 & 312,0989 & $\begin{array}{l}\mathrm{C} 13 \mathrm{H} 17 \mathrm{~N} 4 \\
\mathrm{O} 3 \mathrm{Cl}\end{array}$ & $\begin{array}{l}\text { 2-(4-Chloro-3-nitro-1H-pyrazol-1-yl)-N- } \\
\text { [2-(1-cyclohexen-1-yl)ethyl]acetamide }\end{array}$ \\
\hline 8.738 & $0,4153 \%$ & 357,1571 & 357,1576 & $\begin{array}{l}\mathrm{C} 20 \mathrm{H} 23 \mathrm{NO} \\
5\end{array}$ & $\begin{array}{c}\text { Methyl 4- }\{[(3,4- \\
\text { diethoxyphenyl)acetyl]amino\}benzoate }\end{array}$ \\
\hline 9.218 & $4,0775 \%$ & 230,0939 & 230,0943 & C14H1403 & $\begin{array}{l}\text { 3-Hydroxy-8,9,10,11-tetrahydro-7H- } \\
\text { cyclohepta[c]chromen-6-one }\end{array}$ \\
\hline 9.687 & $3,2395 \%$ & 529,4457 & 529,4455 & $\begin{array}{l}\text { C29H59N3 } \\
\text { 05 }\end{array}$ & UNKNOWN \\
\hline 9.835 & $7,0523 \%$ & 523,199 & 523,1995 & $\begin{array}{l}\mathrm{C} 32 \mathrm{H} 29 \mathrm{NO} \\
6\end{array}$ & $\begin{array}{c}\text { 5-(4-Ethoxyphenyl)-1-(2-furylmethyl)- } \\
\text { 3-hydroxy-4-\{4-[(3- } \\
\text { methylbenzyl)oxy]benzoyl }\}-1,5- \\
\text { dihydro-2H-pyrrol-2-one }\end{array}$ \\
\hline $\begin{array}{c}10.05 \\
2\end{array}$ & $0,2028 \%$ & 895,4073 & 895,4072 & $\begin{array}{l}\text { C27H69N13 } \\
\text { 012S4 }\end{array}$ & UNKNOWN \\
\hline $\begin{array}{c}10.16 \\
7\end{array}$ & $0,4837 \%$ & 270,0893 & 270,0892 & C16H1404 & Diphenyl succinate \\
\hline $\begin{array}{c}10.49 \\
8\end{array}$ & $1,9713 \%$ & 356,09 & 356,0896 & C19H1607 & $\begin{array}{c}\text { Methyl }\{[3 \text {-(4-methoxyphenoxy)-4-oxo- } \\
\text { 4H-chromen-7-yl]oxy\}acetate }\end{array}$ \\
\hline $\begin{array}{c}10.64 \\
7\end{array}$ & $0,1652 \%$ & 288,0994 & 288,0998 & C16H1605 & $\begin{array}{l}\text { 1-(2,4-Dihydroxyphenyl)-2-(3,4- } \\
\text { dimethoxyphenyl)ethanone }\end{array}$ \\
\hline $\begin{array}{c}10.89 \\
9\end{array}$ & $1,9083 \%$ & 497,4555 & 497,4557 & $\begin{array}{l}\text { C29H59N3 } \\
\text { O3 }\end{array}$ & UNKNOWN \\
\hline $\begin{array}{c}11.33 \\
3\end{array}$ & $1,3305 \%$ & 542,1941 & 542,1941 & С32H3008 & $\begin{array}{c}\text { 2-[6-(Benzyloxy)-1,3-benzodioxol-5-yl]- } \\
\text { 1-[4-(benzyloxy)-2-hydroxyphenyl]-3,3- } \\
\text { dimethoxy-1-propanone }\end{array}$ \\
\hline $\begin{array}{c}11.48 \\
2\end{array}$ & $2,7945 \%$ & 481,4609 & 481,4608 & $\begin{array}{l}\text { C29H59N3 } \\
02\end{array}$ & N1,N5-Didodecylglutamamide \\
\hline $\begin{array}{c}11.77 \\
9\end{array}$ & $1,8164 \%$ & 300,1332 & 300,1335 & $\begin{array}{l}\text { C14H16N6 } \\
\text { O2 }\end{array}$ & $\begin{array}{l}\text { 1-(4,6-dimethylpyrimidin-2-yl)-3-(4- } \\
\text { methyl-3-nitrophenyl)guanidine }\end{array}$ \\
\hline $\begin{array}{c}12.17 \\
9\end{array}$ & $0,0549 \%$ & 542,1925 & 542,1927 & $\begin{array}{l}\mathrm{C} 29 \mathrm{H} 22 \mathrm{~N} 10 \\
\mathrm{O} 2\end{array}$ & $\begin{array}{l}\text { 3,3'-(2-Methyl-1,3-phenylene)bis(7- } \\
\text { methyl-8-phenylpyrazolo[5,1- } \\
\text { d][1,2,3,5]tetrazin-4(3H)-one) }\end{array}$ \\
\hline \multicolumn{6}{|c|}{ E. PALMIFOLIA BULB SOUTH BORNEO } \\
\hline Rt & \%Area & $\begin{array}{c}\text { Measured } \\
\text { Mass }\end{array}$ & $\begin{array}{c}\text { Calculated } \\
\text { Mass }\end{array}$ & $\begin{array}{l}\text { Molecular } \\
\text { Formula }\end{array}$ & Nama IUPAC \\
\hline 0.254 & $0,0160 \%$ & 124,9779 & 124,9781 & $\mathrm{C}_{4} \mathrm{~N}_{3} \mathrm{Cl}$ & $\begin{array}{c}\text { Chloromethanetricarbonitrile } \\
\{[3-(2-0 x o-2 H-c h r o m e n-3-y l)-1-\text { phenyl- }\end{array}$ \\
\hline 0.689 & $4,7948 \%$ & 364,0964 & 364,0961 & $\mathrm{C}_{22} \mathrm{H}_{12} \mathrm{~N}_{4} \mathrm{O}_{2}$ & $\begin{array}{c}\text { 1H-pyrazol-4- } \\
\text { yl]methylene\}malononitrile }\end{array}$ \\
\hline 2.781 & $0,0207 \%$ & 124,9781 & 124,9783 & $\mathrm{CH}_{3} \mathrm{NO}_{4} \mathrm{~S}$ & Nitromethanesulfinic acid \\
\hline 3.433 & $0,0342 \%$ & 124,9778 & 124,9783 & $\mathrm{CH}_{3} \mathrm{NO}_{4} \mathrm{~S}$ & $\begin{array}{c}\text { Nitromethanesulfinic acid } \\
\text { 1-[2-(Diethylamino)ethyl]-3-[3- }\end{array}$ \\
\hline 3.650 & $0,0277 \%$ & 431,2717 & 431,2717 & C23H45NS3 & $\begin{array}{c}\text { (dimethylamino)propyl]-1-[(8-methyl- } \\
\text { 2-oxo-1,2-dihydro-3- } \\
\text { quinolinyl)methyl]thiourea } \\
\end{array}$ \\
\hline
\end{tabular}




\begin{tabular}{|c|c|c|c|c|c|}
\hline \multicolumn{6}{|c|}{ E. PALMIFOLIA BULB SOUTH BORNEO } \\
\hline Rt & \%Area & $\begin{array}{l}\text { Measured } \\
\text { Mass }\end{array}$ & $\begin{array}{l}\text { Calculated } \\
\text { Mass }\end{array}$ & $\begin{array}{l}\text { Molecular } \\
\text { Formula }\end{array}$ & Nama IUPAC \\
\hline 3.833 & $0,3806 \%$ & 475,3002 & 475,3006 & $\begin{array}{l}\text { C21H41N5 } \\
07\end{array}$ & 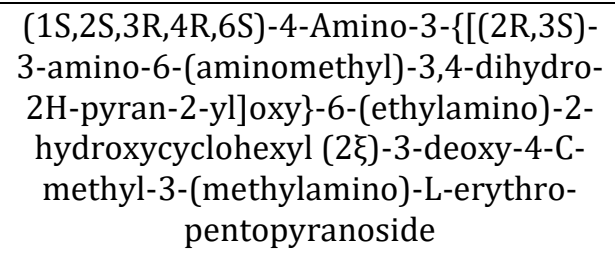 \\
\hline 4.016 & $4,5107 \%$ & 418,1269 & 418,1264 & C21H2209 & $\begin{array}{l}\text { 8,8'-(2-Furylmethylene)bis(6,10- } \\
\text { dioxaspiro[4.5]decane-7,9-dione) }\end{array}$ \\
\hline 4.279 & $4,1290 \%$ & 590,1624 & 590,1624 & $\begin{array}{l}\text { C33H26N4 } \\
\text { O5S }\end{array}$ & $\begin{array}{c}\text { 1-[3-(3,4-Dimethoxyphenyl)-1-phenyl- } \\
\text { 1H-pyrazol-4-yl]-2-(4,5-dimethyl-1,3- } \\
\text { thiazol-2-yl)-1,2-dihydrochromeno[2,3- } \\
\text { c]pyrrole-3,9-dione }\end{array}$ \\
\hline 4.462 & $1,2960 \%$ & 578,3432 & 578,3428 & $\begin{array}{l}\text { C28H46N6 } \\
07\end{array}$ & $\begin{array}{c}\text { L-Phenylalanyl-L-leucyl-L-seryl-N-[(2S)- } \\
\text { 6-amino-1-oxo-2-hexanyl]-L- } \\
\text { threoninamide }\end{array}$ \\
\hline 4.645 & $5,6249 \%$ & 636,2045 & 636,2043 & $\begin{array}{l}\text { C35H32N4 } \\
\text { O6S }\end{array}$ & $\begin{array}{c}\text { 4-[Bis(5-hydroxy-3-methyl-1-phenyl- } \\
\text { 1H-pyrazol-4-yl)methyl]-2- } \\
\text { methoxyphenyl 4- } \\
\text { methylbenzenesulfonate }\end{array}$ \\
\hline 4.827 & $2,9975 \%$ & 620,2097 & 620,2092 & $\begin{array}{l}\mathrm{C} 27 \mathrm{H} 28 \mathrm{~N} 10 \\
08\end{array}$ & $\begin{array}{c}\text { 4-[(E)-(\{[1-(4-Amino-1,2,5-oxadiazol-3- } \\
\text { yl)-4-(4-morpholinylmethyl)-1H-1,2,3- } \\
\text { triazol-5- } \\
\text { yl]carbonyl\}hydrazono)methyl }]-2- \\
\text { ethoxyphenyl 3-methyl-4-nitrobenzoate }\end{array}$ \\
\hline 4.976 & $0,0933 \%$ & 244,0731 & 244,0736 & C14H1204 & 1,4-Naphthalenediyl diacetate \\
\hline 5.159 & $0,2942 \%$ & 520,2071 & 520,2069 & $\begin{array}{l}\mathrm{H} 29 \mathrm{~N} 2905 \mathrm{~S} \\
3\end{array}$ & UNKNOWN \\
\hline 5.628 & $5,6724 \%$ & 256,0726 & 256,0727 & $\begin{array}{l}\text { C7H16N20 } \\
6 \mathrm{~S}\end{array}$ & $\begin{array}{l}\text { 2-Amino-N-isopropylethanesulfonamide } \\
\text { ethanedioate }(1: 1)\end{array}$ \\
\hline 5.891 & $\begin{array}{c}13,2610 \\
\%\end{array}$ & 272,1042 & 272,1042 & $\begin{array}{l}\text { C8H20N2O } \\
6 \mathrm{~S}\end{array}$ & Morpholine sulfate $(2: 1)$ \\
\hline 6.508 & $0,3749 \%$ & 242,0575 & 242,0573 & $\begin{array}{l}\text { C6H14N2O } \\
6 \mathrm{~S}\end{array}$ & $\begin{array}{l}\text { 6-Deoxy-N-sulfamoyl- } \beta \text {-D- } \\
\text { mannopyranosylamine }\end{array}$ \\
\hline 6.805 & $0,0461 \%$ & 242,0569 & 242,0571 & $\begin{array}{l}\mathrm{C} 9 \mathrm{H} 11 \mathrm{~N} 40 \\
2 \mathrm{Cl}\end{array}$ & $\begin{array}{l}\text { 7-(2-Chloroethyl)-1,3-dimethyl-3,7- } \\
\text { dihydro-1H-purine-2,6-dione }\end{array}$ \\
\hline 6.988 & $2,9684 \%$ & 244,0741 & 244,0736 & C14H1204 & $\begin{array}{c}\text { 4-[(E)-2-(3,5-Dihydroxyphenyl)vinyl }]- \\
\text { 1,3-benzenediol } \\
\text { (Oxyresveratrol) }\end{array}$ \\
\hline 7.354 & $0,5432 \%$ & 258,0879 & 258,0884 & $\begin{array}{l}\mathrm{C} 10 \mathrm{H} 15 \mathrm{~N} 4 \\
\mathrm{O} 2 \mathrm{Cl}\end{array}$ & $\begin{array}{l}\text { 3-Nitro-N-(4-piperidinyl)-2- } \\
\text { pyridinamine hydrochloride }(1: 1)\end{array}$ \\
\hline 7.640 & $8,0227 \%$ & 258,1471 & 258,1467 & C13H2205 & $\begin{array}{l}\text { tert-Butyl 3,5-dideoxy-2,4-0- } \\
\text { isopropylidene-L-erythro-hexuronate }\end{array}$ \\
\hline 7.903 & $4,9125 \%$ & 272,1047 & 272,1049 & C16H1604 & $\begin{array}{l}\text { 2-Hydroxy-1,2-bis(4- } \\
\text { methoxyphenyl)ethanone }\end{array}$ \\
\hline 8.086 & $5,8744 \%$ & 228,0782 & 228,0787 & C14H12O3 & $\begin{array}{c}\text { 5-[(E)-2-(4-Hydroxyphenyl)vinyl }]-1,3- \\
\text { benzenediol } \\
\text { (Trans-resveratrol) }\end{array}$ \\
\hline 8.406 & $\begin{array}{c}26,4818 \\
\%\end{array}$ & 244,073 & 244,0729 & $\begin{array}{l}\text { C6H16N2O } \\
6 \mathrm{~S} \\
\end{array}$ & Lysine sulfate $(1: 1)$ \\
\hline
\end{tabular}




\begin{tabular}{|c|c|c|c|c|c|}
\hline \multicolumn{6}{|c|}{ E. PALMIFOLIA BULB SOUTH BORNEO } \\
\hline Rt & $\%$ Area & $\begin{array}{l}\text { Measured } \\
\text { Mass }\end{array}$ & $\begin{array}{l}\text { Calculated } \\
\text { Mass }\end{array}$ & $\begin{array}{l}\text { Molecular } \\
\text { Formula }\end{array}$ & Nama IUPAC \\
\hline 9.104 & $1,2478 \%$ & 723,3323 & 723,3327 & $\begin{array}{l}\text { C33H49N5 } \\
013\end{array}$ & $\begin{array}{c}\text { (2R,3R,4R,5S)-5-[(1R)-2-Amino-1- } \\
\{[(2 \mathrm{~S}, 3 \mathrm{~S}, 4 \mathrm{~S})-3,4-\text { dihydroxy-6- }\{[(3 \mathrm{~S})-2- \\
\text { oxo-3-azepanyl]carbamoyl }\}-3,4- \\
\text { dihydro-2H-pyran-2-yl]oxy }\}-2- \\
\text { oxoethyl }]-2-(2,4-\text { dioxo-3,4-dihydro- } \\
\text { 1(2H)-pyrimidinyl)-4-methoxytetrahyd } \\
\text { ro-3-furanyl decanoate }\end{array}$ \\
\hline 9.218 & $0,3255 \%$ & 526,1629 & 526,1628 & C31H2608 & $\begin{array}{c}\text { 4-(\{[3-(2,3-Dihydro-1,4-benzodioxin-6- } \\
\text { yl)-4-oxo-6-propyl-4H-chromen-7- } \\
\text { yl]oxy\}methyl)-7-methoxy-2H- } \\
\text { chromen-2-one }\end{array}$ \\
\hline 9.366 & $0,0481 \%$ & 468,139 & 468,1389 & $\begin{array}{l}\text { C21H28N2 } \\
\text { 06S2 }\end{array}$ & $\begin{array}{c}\text { 1,4-Bis[(4-methoxy-3- } \\
\text { methylphenyl)sulfonyl]-1,4-diazepane }\end{array}$ \\
\hline 9.584 & $1,0705 \%$ & 809,3669 & 809,3668 & $\begin{array}{l}\text { C33H51N11 } \\
013\end{array}$ & $\begin{array}{l}\text { Glycyl-L-seryl-N5-(diaminomethylene)- } \\
\text { L-ornithyl-L-glutaminyl-L-tyrosyl-L- } \\
\text { alanyl-L-glutamic acid }\end{array}$ \\
\hline 9.732 & $0,8358 \%$ & 169,0883 & 169,0885 & $\begin{array}{c}\mathrm{C} 4 \mathrm{H} 15 \mathrm{~N} 30 \\
2 \mathrm{~S}\end{array}$ & UNKNOWN \\
\hline $\begin{array}{c}10.05 \\
2\end{array}$ & $0,7553 \%$ & 895,4057 & 895,4056 & $\begin{array}{l}\text { C31H53N21 } \\
\text { O9S }\end{array}$ & UNKNOWN \\
\hline $\begin{array}{c}10.16 \\
7\end{array}$ & $0,1860 \%$ & 270,0892 & 270,0892 & C16H1404 & Diphenyl succinate \\
\hline $\begin{array}{c}10.35 \\
0\end{array}$ & $0,5501 \%$ & 513,4505 & 513,4506 & $\begin{array}{l}\text { C29H59N3 } \\
04\end{array}$ & UNKNOWN \\
\hline $\begin{array}{c}10.49 \\
8\end{array}$ & $1,6500 \%$ & 356,0891 & 356,0896 & C19H1607 & $\begin{array}{c}\text { Methyl }\{[3-(4-m e t h o x y p h e n o x y)-4-o x o- \\
\text { 4H-chromen-7-yl] oxy }\} \text { acetate }\end{array}$ \\
\hline $\begin{array}{c}10.68 \\
1\end{array}$ & $0,2252 \%$ & 298,0834 & 298,0835 & $\begin{array}{l}\text { C9H18N2O } \\
7 \mathrm{~S}\end{array}$ & $\begin{array}{c}2-[(2-\{[(3- \\
\text { Aminopropyl)carbamoyl]oxy\}ethyl)sulf } \\
\text { onyl] ethyl hydrogen carbonate }\end{array}$ \\
\hline $\begin{array}{c}10.78 \\
4 \\
\end{array}$ & $0,0886 \%$ & $\begin{array}{c}1067,476 \\
1 \\
\end{array}$ & 1067,4761 & $\begin{array}{l}\text { C73H65NO } \\
7 \\
\end{array}$ & UNKNOWN \\
\hline \multicolumn{6}{|c|}{ E. PALMIFOLIA BULB CENTRAL JAVA } \\
\hline Rt & \%Area & $\begin{array}{l}\text { Measured } \\
\text { Mass }\end{array}$ & $\begin{array}{l}\text { Calculated } \\
\text { Mass }\end{array}$ & $\begin{array}{l}\text { Rumus } \\
\text { Molekul }\end{array}$ & Nama IUPAC \\
\hline 0,689 & $0,9161 \%$ & 380,0728 & 380,0726 & $\begin{array}{l}\mathrm{C}_{14} \mathrm{H}_{16} \mathrm{~N}_{6} \mathrm{O}_{3} \mathrm{~S} \\
2\end{array}$ & $\begin{array}{c}\text { 2-(\{[2-(Methoxymethyl)-5-oxo-5H- } \\
{[1,3,4] \text { thiadiazolo[3,2-a]pyrimidin-7- }} \\
\text { yl]methyl }\} \text { sulfanyl)-N-(1-methyl-1H- } \\
\text { pyrazol-4-yl)acetamide }\end{array}$ \\
\hline 1,020 & $0,1982 \%$ & 293,1478 & 293,1475 & $\mathrm{C}_{12} \mathrm{H}_{23} \mathrm{NO}_{7}$ & $\begin{array}{l}\text { 1,2-di-0-methyl-4-[(2R)-2,4- } \\
\text { dihydrobutyramido]-4,6-dideoxy- } \alpha-\mathrm{D}- \\
\text { mannopyranoside }\end{array}$ \\
\hline 1,386 & $0,0434 \%$ & 327,1317 & 327,1318 & $\mathrm{C}_{15} \mathrm{H}_{21} \mathrm{NO}_{7}$ & $\begin{array}{l}\text { Methyl (3,4,5-triethoxy-2- } \\
\text { nitrophenyl)acetate }\end{array}$ \\
\hline 2,598 & $0,0034 \%$ & 181,9777 & 181,9778 & $\mathrm{CH}_{3} \mathrm{~N}_{6} \mathrm{OSCl}$ & UNKNOWN \\
\hline 2,735 & $0,0046 \%$ & 181,9778 & 181,9778 & $\mathrm{CH}_{3} \mathrm{~N}_{6} \mathrm{OSCl}$ & UNKNOWN \\
\hline 3,250 & $0,1746 \%$ & 230,1055 & 230,1056 & $\mathrm{C}_{13} \mathrm{H}_{14} \mathrm{~N}_{2} \mathrm{O}_{2}$ & $\begin{array}{l}\text { Ethyl 5-methyl-1-phenyl-1H-pyrazole-4- } \\
\text { carboxylate }\end{array}$ \\
\hline 3,696 & $0,0679 \%$ & 292,0589 & 292,0588 & $\mathrm{C}_{10} \mathrm{H}_{9} \mathrm{~N}_{8} \mathrm{OCl}$ & $\begin{array}{c}\text { 4-Chloro-N-(4-imino-1,4-dihydro-5H- } \\
\text { pyrazolo[3,4-d]pyrimidin-5-yl)-1- } \\
\text { methyl-1H-pyrazole-5-carboxamide }\end{array}$ \\
\hline
\end{tabular}




\begin{tabular}{|c|c|c|c|c|c|}
\hline \multicolumn{6}{|c|}{ E. PALMIFOLIA BULB CENTRAL JAVA } \\
\hline Rt & \%Area & $\begin{array}{l}\text { Measured } \\
\text { Mass }\end{array}$ & $\begin{array}{l}\text { Calculated } \\
\text { Mass }\end{array}$ & $\begin{array}{c}\text { Rumus } \\
\text { Molekul }\end{array}$ & Nama IUPAC \\
\hline 4,565 & $1,1948 \%$ & 244,0736 & 244,0736 & C14H12O4 & 1,4-Naphthalenediyl diacetate \\
\hline 4,976 & $0,5442 \%$ & 596,2099 & 596,2094 & $\begin{array}{c}\text { C18H36N12 } \\
\text { O5S3 }\end{array}$ & UNKNOWN \\
\hline 5,113 & $1,1116 \%$ & 596,2119 & 596,2119 & $\begin{array}{l}\text { C29H32N4 } \\
010\end{array}$ & $\begin{array}{l}\text { (4aR,7aS,7bS,8R,9R)-9a-Acetoxy-4a,7b- } \\
\text { dihydroxy-3-(hydroxymethyl)-1,1,6,8- } \\
\text { tetramethyl-5-oxo- } \\
\text { 1a,1b,4,4a,5,7a,7b,8,9,9a-decahydro-1H- } \\
\text { cyclopropa[3,4]benzo[1,2-e]azulen-9-yl } \\
\text { 5-azido-2-nitrobenzoate }\end{array}$ \\
\hline 5,708 & $1,1017 \%$ & 256,1095 & 256,1100 & C16H16O3 & $\begin{array}{l}\text { 4-(Benzyloxy)-3-ethoxybenzaldehyde } \\
\text { (2E)-1-(2,4-Dihydroxyphenyl)-3-(4- }\end{array}$ \\
\hline 5,891 & $0,0978 \%$ & 256,0738 & 256,0736 & C15H1204 & $\begin{array}{l}\text { hydroxyphenyl)-2-propen-1-one } \\
\text { (Isoliquiritigenin) }\end{array}$ \\
\hline 6,028 & $0,4945 \%$ & 256,0744 & 256,0749 & C16H8N4 & $\begin{array}{c}\text { Quinoxalino[2',3':3,4]cyclobuta[1,2- } \\
\text { b]quinoxaline }\end{array}$ \\
\hline 6,325 & $0,1239 \%$ & 274,085 & 274,0855 & $\begin{array}{l}\mathrm{C} 16 \mathrm{H} 10 \mathrm{~N} 4 \\
\mathrm{O}\end{array}$ & $\begin{array}{l}\text { 4,7-Diphenyl }(1,2,5) \text { oxadiazolo }(3,4- \\
\text { d)pyridazine }\end{array}$ \\
\hline 6,577 & $0,3646 \%$ & 287,116 & 287,1158 & $\begin{array}{l}\text { C16H17NO } \\
4\end{array}$ & $\begin{array}{l}\text { Methyl 5-(1-acetamido-2-phenylethyl)- } \\
\text { 2-furoate }\end{array}$ \\
\hline 7,389 & $6,2525 \%$ & 244,0737 & 244,0736 & C14H1204 & $\begin{array}{c}\text { 4-[(E)-2-(3,5-Dihydroxyphenyl)vinyl }]- \\
\text { 1,3-benzenediol } \\
\text { (Oxyresveratrol) }\end{array}$ \\
\hline 7,606 & $0,2237 \%$ & 370,2833 & 370,2832 & $\begin{array}{c}\mathrm{C} 20 \mathrm{H} 38 \mathrm{~N} 2 \\
\mathrm{O} 4\end{array}$ & UNKNOWN \\
\hline 8,452 & $8,4865 \%$ & 228,0789 & 228,0787 & C14H12O3 & $\begin{array}{c}\text { 5-[(E)-2-(4-Hydroxyphenyl)vinyl }]-1,3- \\
\text { benzenediol } \\
\text { (Trans-resveratrol) }\end{array}$ \\
\hline 8,738 & $\begin{array}{c}13,3947 \\
\%\end{array}$ & 244,0738 & 244,0736 & C14H12O4 & 1,4-Naphthalenediyl diacetate \\
\hline 8,955 & $1,8921 \%$ & 312,1003 & 312,0998 & C18H1605 & $\begin{array}{c}\text { Isopropyl [(6-oxo-6H-benzo[c]chromen- } \\
3-y l) o x y] \text { acetate }\end{array}$ \\
\hline 9,321 & $0,1779 \%$ & 270,0897 & 270,0892 & C16H1404 & Diphenyl succinate \\
\hline 9,652 & $1,0856 \%$ & 371,1734 & 371,1733 & $\begin{array}{l}\mathrm{C} 21 \mathrm{H} 25 \mathrm{NO} \\
5\end{array}$ & $\begin{array}{c}\text { 1,2,3,10-Tetramethoxy-7- } \\
\text { (methylamino)-6,7- } \\
\text { dihydrobenzo[a]heptalen-9(5H)-one }\end{array}$ \\
\hline $\begin{array}{c}10.05 \\
3\end{array}$ & $3,3308 \%$ & 315,2772 & 315,2774 & $\begin{array}{l}\text { C18H37NO } \\
3\end{array}$ & $\begin{array}{l}\text { N,N-Bis(2- } \\
\text { hydroxyethyl)tetradecanamide }\end{array}$ \\
\hline $\begin{array}{c}10.23 \\
5\end{array}$ & $9,9337 \%$ & 529,4459 & 529,4455 & $\begin{array}{c}\text { C29H59N3 } \\
05\end{array}$ & UNKNOWN \\
\hline $\begin{array}{c}10.38 \\
4\end{array}$ & $6,9546 \%$ & 523,2003 & 523,2000 & $\begin{array}{l}\text { C17H25N13 } \\
07\end{array}$ & UNKNOWN \\
\hline $\begin{array}{c}11.15 \\
0\end{array}$ & $2,9977 \%$ & 258,1255 & 258,1256 & C16H1903 & 5-(Adamantan-1-yl)-2-methyl-3-furoate \\
\hline $\begin{array}{l}11.44 \\
7\end{array}$ & $\begin{array}{c}10,4767 \\
\%\end{array}$ & 497,4562 & 497,4557 & $\begin{array}{l}\text { C29H60N3 } \\
\text { O3 }\end{array}$ & UNKNOWN \\
\hline $\begin{array}{c}11.81 \\
3\end{array}$ & $3,9633 \%$ & 541,4823 & 541,4819 & $\begin{array}{c}\text { C31H63N3 } \\
04\end{array}$ & UNKNOWN \\
\hline $\begin{array}{c}12.17 \\
9\end{array}$ & $0,5320 \%$ & 466,3089 & 466,3086 & $\begin{array}{l}\text { C23H50N2 } \\
\text { OS3 }\end{array}$ & UNKNOWN \\
\hline $\begin{array}{c}12.39 \\
6\end{array}$ & $1,0792 \%$ & 341,3295 & 341,3294 & $\begin{array}{c}\mathrm{C} 21 \mathrm{H} 43 \mathrm{NO} \\
2\end{array}$ & Ethyl N-hexadecyl- $\beta$-alaninate \\
\hline
\end{tabular}


Metabolite Fingerprinting Eleutherine palmifolia (L.) Merr.

\begin{tabular}{|c|c|c|c|c|c|}
\hline \multicolumn{6}{|c|}{ E. PALMIFOLIA BULB CENTRAL JAVA } \\
\hline Rt & \%Area & $\begin{array}{c}\text { Measured } \\
\text { Mass }\end{array}$ & $\begin{array}{c}\text { Calculated } \\
\text { Mass }\end{array}$ & $\begin{array}{c}\text { Rumus } \\
\text { Molekul }\end{array}$ & Nama IUPAC \\
\hline $\begin{array}{c}12.54 \\
5\end{array}$ & $1,1901 \%$ & 468,3241 & 468,3240 & $\mathrm{C} 30 \mathrm{H} 4404$ & $\begin{array}{l}(5 \xi, 9 \xi)-3,11-\text { Dioxoolean-12-en-30-oic } \\
\text { acid }\end{array}$ \\
\hline $\begin{array}{c}12.76 \\
2\end{array}$ & $2,8968 \%$ & 399,2408 & 399,2410 & $\begin{array}{c}\mathrm{C} 24 \mathrm{H} 33 \mathrm{NO} \\
4\end{array}$ & $\begin{array}{c}\mathrm{N}-(3,4-\text { Dimethoxybenzyl)-N-[3-(4- } \\
\text { methoxyphenyl)-4- } \\
\text { methylpentyl]acetamide }\end{array}$ \\
\hline $\begin{array}{c}12.87 \\
7\end{array}$ & $3,7969 \%$ & 323,2824 & 323,2825 & $\begin{array}{c}\mathrm{C} 20 \mathrm{H} 37 \mathrm{NO} \\
2\end{array}$ & $\begin{array}{c}\text { 1-[(2R)-1,4-Dioxaspiro[4.11]hexadec-2- } \\
\text { ylmethyl]piperidine } \\
\text { N-[3-(4-Isonronvl-2 } 2 \text { - }\end{array}$ \\
\hline $\begin{array}{c}13.16 \\
2\end{array}$ & $8,7991 \%$ & 441,2519 & 441,2515 & $\begin{array}{c}\mathrm{C} 26 \mathrm{H} 35 \mathrm{NO} \\
5\end{array}$ & $\begin{array}{c}\text { dimethyltetrahydro-2H-pyran-4- } \\
\text { yl)propanoyl]-N-(4-methoxybenzyl)-2- } \\
\text { furamide }\end{array}$ \\
\hline $\begin{array}{c}13.31 \\
1\end{array}$ & $0,8928 \%$ & 523,3642 & 523,3640 & $\begin{array}{c}\text { C14H41N19 } \\
\text { O3 }\end{array}$ & UNKNOWN \\
\hline $\begin{array}{c}13.46 \\
0\end{array}$ & $2,2781 \%$ & 299,2828 & 299,2825 & $\begin{array}{l}\mathrm{C} 18 \mathrm{H} 37 \mathrm{NO} \\
2\end{array}$ & N-(2-Hydroxyethyl)hexadecanamide \\
\hline $\begin{array}{c}13.60 \\
8\end{array}$ & $1,5235 \%$ & 278,225 & 278,2246 & C18H30O2 & $\begin{array}{l}\text { (9E,12E,15E)-9,12,15- } \\
\text { Octadecatrienoic acid }\end{array}$ \\
\hline $\begin{array}{c}13.79 \\
1\end{array}$ & $1,4004 \%$ & 759,4714 & 759,4710 & $\begin{array}{l}\text { C46H65NO } \\
8\end{array}$ & $\begin{array}{l}3-[\{6-[3,5- \\
\text { Bis(decyloxy)phenoxy]hexanoyl }\}(4- \\
\text { carboxyphenyl)amino]benzoic acid }\end{array}$ \\
\hline
\end{tabular}

Java sample, 34 compounds from the East Borneo sample, 38 compounds from the Central Borneo sample, and 31 compounds from the South Borneo sample. The composition of the compounds in plants influenced by two factors, namely internal factors and external factors (Heuberger et al., 2013). Internal factors that affect the composition of the compound include genetic and physiological variations, while external factors such as geographical conditions (altitude), climate, humidity, light intensity, temperature, nutrient intake, and radiation (Verma and Shukla, 2015). These factors cause the number of compounds from each sample to be different.

Table IV shows the different types of compounds contained in E. palmifolia bulbs from six different growing locations. Each sample $E$. palmifolia bulb showed the presence of a dominant or major compound, including $N$ - (ethylcarbamoyl) -2 - [(1-methyl-1H-tetrazole-5-yl) sulfanyl] acetamide with a percent area of $25.928 \%$ at samples from Blitar, East Java; 1,4-Naphthalenediyl diacetate with a percent area of 13,395\% in samples originating from Karanganyar, Central Java; Lysine sulfate (1: 1) with a percent area of $24.638 \%$ in samples originating from Bogor, West Java; $N$ - (ethylcarbamoyl) -2 - [(1-methyl-1Htetrazole-5-yl) sulfanyl] acetamide with a percentage area of $24.457 \%$ in samples from Balikpapan, East Borneo; Lysine sulfate (1: 1) with a percentage area of $27,180 \%$ in samples originating from West Kotawaringin, Central Borneo; and Lysine sulfate (1: 1) with an area of $26.48 \%$ in samples from Banjarmasin, South Borneo.

Based on these data it is also known that $E$. palmifolia bulb contains the compound isoliquiritigenin and resveratrol. Previous studies reported that isoliquiritigenin could inhibit the growth of breast cancer cells and inhibit neoangiogenesis and suppress the growth of colon cancer cells in induced mice azoxymethane (AOM) (Takahashi et al., 2004; Wang et al., 2013). The results of the analysis showed that isoliquiritigenin contained in the sample E. palmifolia bulb originating from East Java. With a retention time of 9,608; Central Java with a retention time of 5.89; West Java with a retention time of 6.074; East Borneo with a retention time of 5.89; and Central Borneo with a retention time of 5.594; in samples from South Borneo no compounds were isoliquiritigenin found. MS spectra of compounds Isoliquiritigenin presented in Figure 2.

As a result of the spectral analysis have also been found two types of resveratrol in samples E.palmifolia studied were bulb Trans-resveratrol and Oxyresveratrol. Trans-resveratrol found in E. palmifolia bulb samples as samples originating from West Java with a retention time of 8,452 ; Central Java with a retention time of 8,452; East Borneo with a retention time of 8,8086 ; and South Borneo with a retention time of 5,452 . In samples 


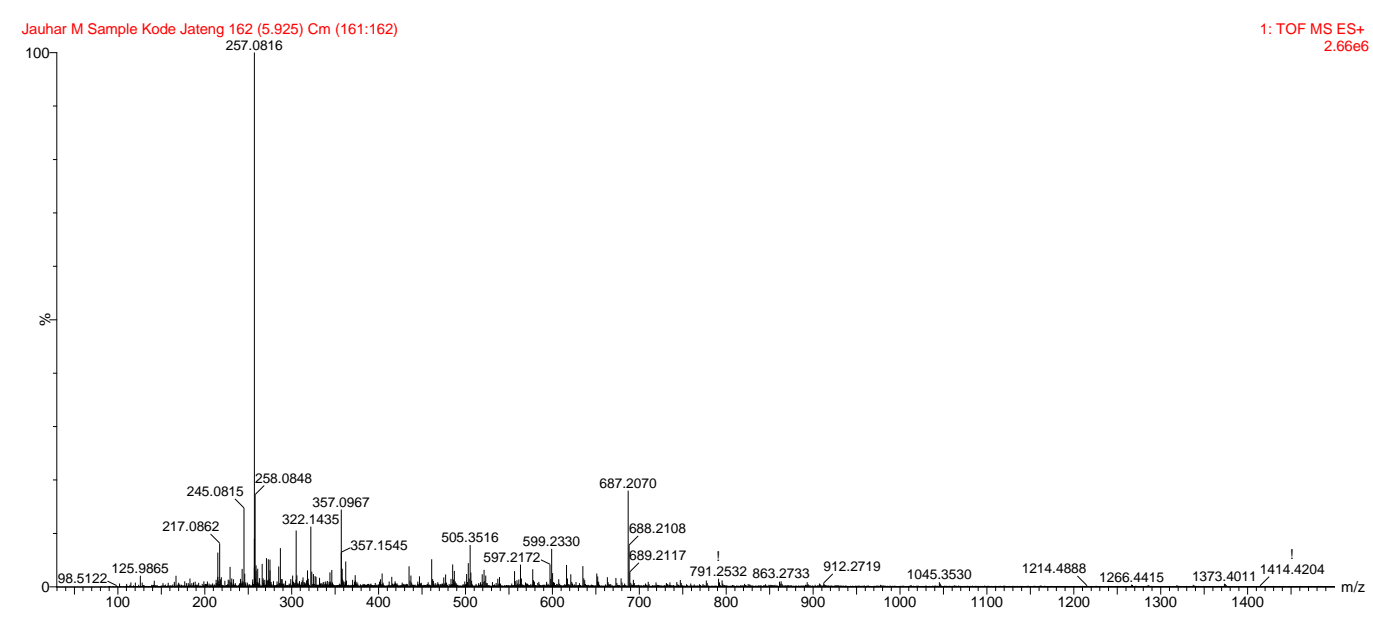

Figure 2. The spectra and structure Compound Isoliquiritigenin $\left(\mathrm{C}_{15} \mathrm{H}_{12} \mathrm{O} 4\right)$.

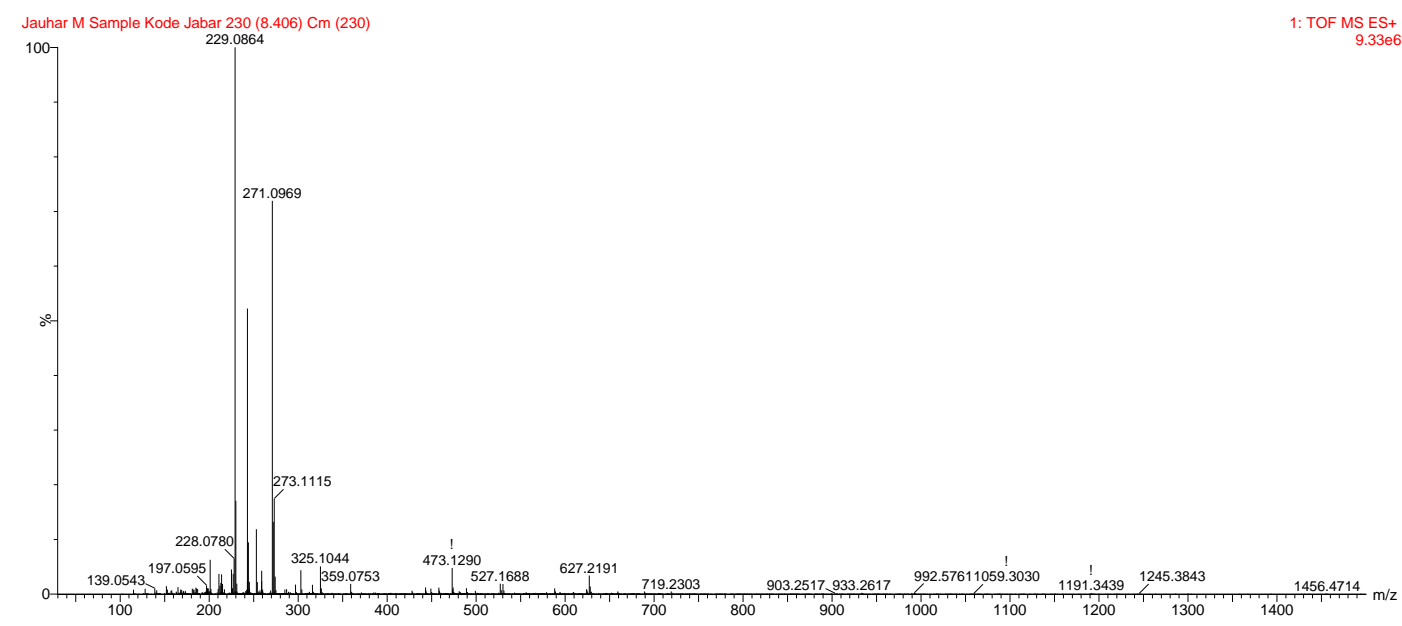

Figure 3. Spectra and structure of compounds Trans-resveratrol $\left(\mathrm{C}_{14} \mathrm{H}_{12} \mathrm{O}_{3}\right)$.

from East Java and Central Borneo, no compounds were found trans-resveratrol. Oxyresveratrol was found in samples E. palmifolia bulb from Central Java with a retention time of 7.39; West Java with a retention time of 6,943 ; Central Borneo with a retention time of 6,988; and South Borneo with a retention time of 6,988 . In samples originating from East Java and Central Borneo, no compounds were oxyresveratrol found.

Resveratrol is phytoalexin in the skin of grapes, nuts, and some fruits. It is known for its effects as an antioxidant and anti-inflammatory and inhibits the proliferation of cancer cells (Smoliga et al., 2011). Resveratrol has successfully inhibited the growth of tumors tested in vivo using several types of cancer, besides that in preclinical studies resveratrol has been shown to improve heart health, reduce blood pressure, and lower blood glucose levels (Singh et al., 2014).
The spectra of trans-resveratrol and oxyresveratrol MS presented in Figures 3 and 4.

\section{Analysis PCA Multivariate Data Using Minitab}

In simple terms, PCA is a linear transformation to determine the new coordinate system of a dataset. This PCA technique reduces or reduces information on extensive data to be simpler without removing existing information (Syakhala et al., 2015). PCA visualization results can be displayed through score plots and loading plots. Score plots describe the closeness between objects (samples), while loading plots describe relationships between variables, namely original variables and new variables (Taufik, 2017). The results of multivariate PCA data analysis E. palmifolia bulb from several locations are presented in Figures 5 and six as follows. 


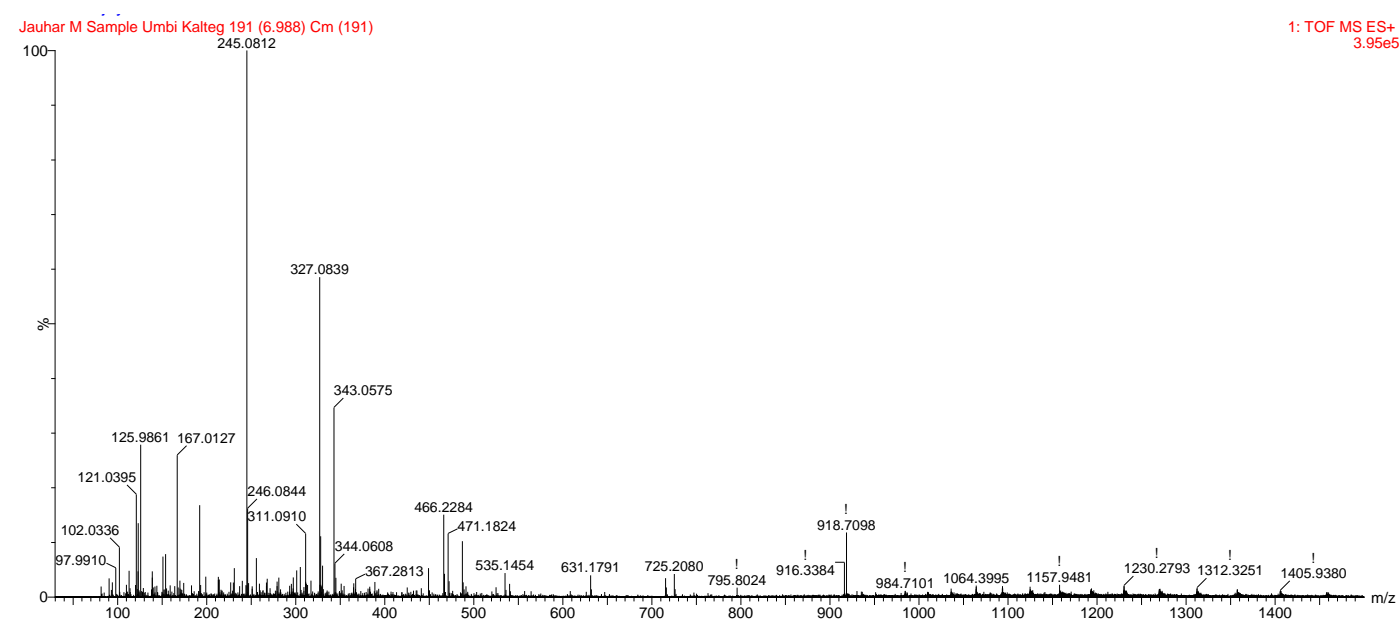

Figure 4. Spectra and structure of compounds Oxyresveratrol $\left(\mathrm{C}_{14} \mathrm{H}_{12} \mathrm{O}_{4}\right)$.

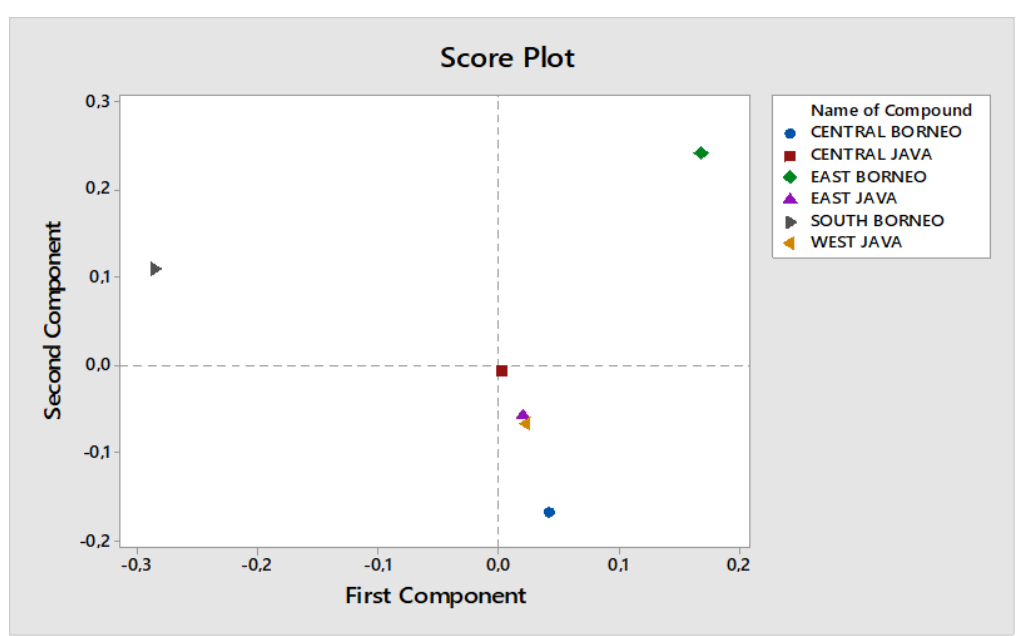

Figure 5. Score Plot That Shows The Similarity Of Types Of Compounds From Blitar, East Java With Bogor, West Java.

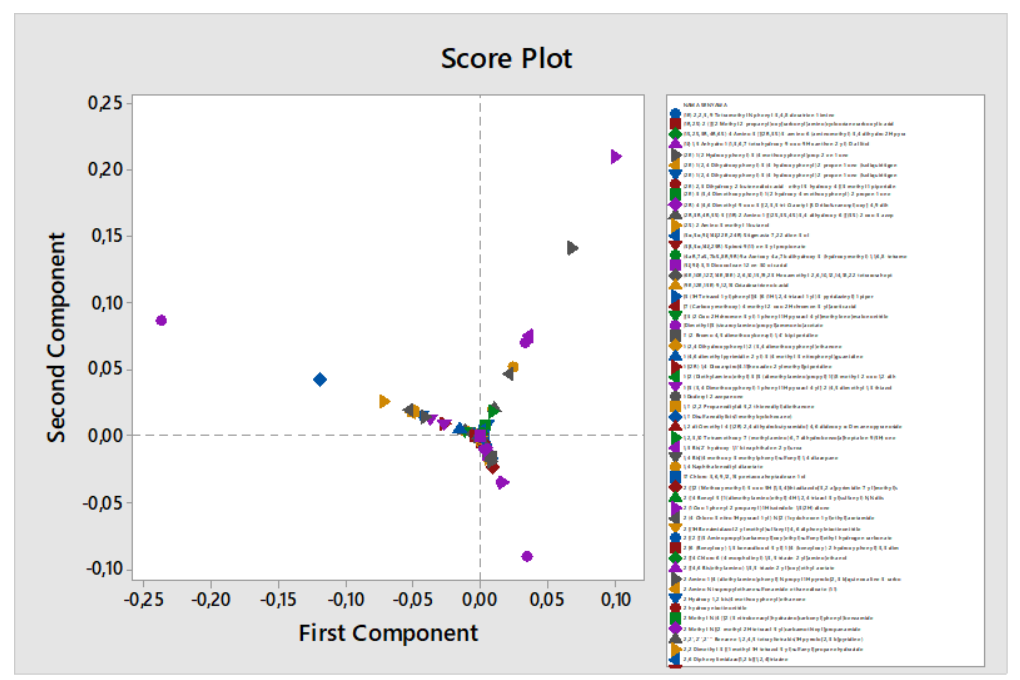

Figure 6. Score plot that shows the existence of compounding groups from the six regions. 


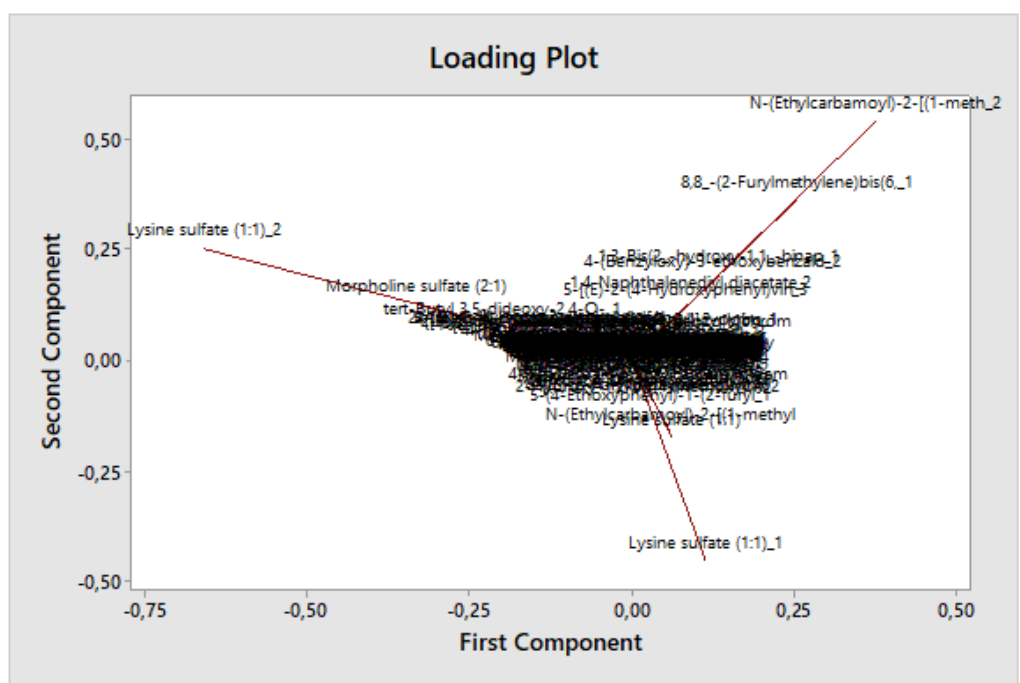

Figure 7. Loading plot that shows several major compounds.

The loading plot in Figure 7 shows the representative compounds of E. palmifolia bulbs originating from several regions in Indonesia. There are at least three compounds, namely Lysine sulfate (1: 1) yang derived from of sample West Java, Central Borneo and South Borneo; $\mathrm{N}$ (ethylcarbamoyl) -2 - [(1-methyl-1H-tetrazole-5-yl) sulfanyl] acetamide obtained from samples from East Java, West Java, and East Borneo; and 8.8'- (2Furyl methylene) bis (6,10-dioxaspiro [4.5] decane7,9-dione) originating from samples from West Java, East Borneo, Central Borneo and South Borneo. One of the three compounds, Lysine sulfate, has excellent benefits for the body. Lysine which is a group of amino acids has an essential role in the immune system because it functions to develop antibodies. The scientists found that Lysine has antiviral activity, increases the effectiveness of L-arginine in promoting the release of human growth hormone $(\mathrm{HGH})$, and can help reduce excessive anxiety (Smriga et al, 2007; Suminski et al., 1997).

Several factors cause the compound content in plants to be similar. These factors can be classified into two, namely internal factors such as genetic and physiological variations, as well as external factors such as geographical conditions (altitude), climate, humidity, light intensity, temperature, nutrient intake, and radiation (Verma N, Shukla S, 2015). The area Blitar, East Java with Karanganyar, Central Java, has a similar content of compounds thought to be caused by external factors. Previously in table I, it was explained about the environmental conditions consisting of altitude, average temperature, rainfall, climate, and soil type of all samples, it is known that the Blitar area, East Java with Bogor, West Java has an average temperature equation $\left({ }^{\circ} \mathrm{C}\right)$ and kind of soil, namely regosol.

\section{CONCLUSION}

The results showed that there were differences in the content of the metabolite compounds in bulb E. palmifolia originating from six different regions. There were 40 compounds from the East Java sample, 32 compounds from the Central Java sample, 39 compounds from the West Java sample, 34 compounds from the East Borneo sample, 38 compounds from the Central Borneo sample, and 31 compounds from the South Borneo sample, some major compounds found were isoliquiritigenin, trans-resveratrol, and oxyresveratrol.

\section{REFERENCES}

Anissa. 2012. Kajian Metabolomik Rimpang Kunyit Menggunakan Kromatografi CairSpektroskopi Massa [Skripsi], Departemen Kimia Institut Pertanian Bogor: Bogor.

Aulia, Savira S., Iyan S, Muchtaridi. Penetapan Kadar Simvastatin Menggunakan Kromatorafi Cair Kinerja Tinggi (Kckt): Review, Farmaka, 14(4):1-8.

Ayunda RD. 2014. Aktivitas Antioksidan Ekstrak Etanol Daun Serai (Cymbopogon citratus) dan Potensinya sebagai Pencegah Oksidasi Lipid [Skripsi]. Departemen Biokimia FMIPA, Institut Pertanian Bogor: Bogor.

Brenton AG, Godfrey AR. 2010. Accurate Mass Measurement: Terminology and Treatment of Data, Journal of American Society for Mass Spectrometry, 21:1821-1835. 
Distantina S, Fadhila YC, Danarto W, Fahrurrozi M. 2009. Pengaruh Kondisi Proses pada Pengolahan Euchema cottoni terhadap Rendemen dan Sifat Gel Karagenan, Ekuilibrium, 8(1): 35-40.

Firdaus, R., 2006, Telaah Kandungan Kimia Ekstrak Metanol Umbi Bawang Tiwai (Eleutherine americana (Aubl.) Merr.) [Skripsi], Institut Teknologi Bandung: Bandung.

Handayani H, Sriherfyna FH, Yunianta. 2016. Ekstraksi Antioksidan Daun Sirsak Metode Ultrasounic Bath (Kajian Rasio Bahan: Pelarut dan Lama Ekstraksi). Jurnal Pangan dan Agroindustri, 4 (1): 262- 272.

Hara H, Naoki M, Shinsuke Y, Yasuhisa H, Kuo-HL, Kenneth FB, Ruji M,Yasuhiro I. 1997. Elecanacin, a Novel New Naphthoquinone from the Bulb of Eleutherine americana, Journal Chem, Pharm, Bull, Pharmaceutical Society of Japan, 45 (10):1-8.

Heuberger, A.l.; Corey DB, Kaylyn R. Kirkpatrick, Jessica EP. 2013. Application of Nontargeted Metabolite Profiling to Discover Novel Markers of Quality Traits in an Advanced Population of Malting Barley, Plant Biotechnology Journal, 12, pp. 147-160.

Ieyama T, Maria DPT, Jun K. 2011. $\alpha$-Glucosidase Inhibitors from the Bulb of Eleutherine americana, Food Chem, 128: 308-311.

Rohman, A. 2009. Kromatografi untuk Analisis Obat (Edisi I). Graha Ilmu: Yogyakarta.

Singh, Chandra K., Ndiaye, Mary A., Ahmad, Nihal, 2014, Resveratrol and Cancer: Challenges for Clinical Translation. BBA - Molecular Basis of Disease.

Skoog DA, Holler FJ, Crouch SR. 2004. Fundamentals of Analytical Chemistry (eighth edition), Thomson Brooks/Cole: Belmont.

Smoliga J.M., Baur J.A., and Hausenblas H.A., 2011, Resveratrol and health - a comprehensive review of human clinical trials, Mol Nutr Food Res, 55: 1129-1141.

Smriga M, Ando T, Akutsu M, Furukawa Y, Miwa K., Morinaga Y., 2007, Oral treatment with Llysine and L-arginine reduces anxiety and basal cortisol levels in healthy humans. Biomedical Research (Tokyo, Japan), 28(2):85-90.

Srivastava M, Bashir AA, Shishir KG, and Shailendra KG. 2010. Development of Resistance
Against Blackleg Disease in Brassica Oleracea var. Botrytis Through in Silico Methods, Fungal Genetics and Biology, 47:800-808.

Suminski R.R. Robertson R.J., Goss F.L., Arslanian S., Kang J., Da Silva S., Utter A.C., and Metz K.F., 1997, Acute Effect of Amino Acid Ingestion and Resistance Exercise on Plasma Growth Hormone Concentration in Young Men. International Journal of Sports Nutrition, 7(1):48-60.

Syakhala, Abdu R., Puspitaningrum, D, Purwandari, Endina P. 2015, Perbandingan Metode Principal Component Analysis (PCA) dengan Metode Hidden Markov Model (HMM) dalam Pengenalan Identitas Seseorang Melalui Wajah, Jurnal Rekursif, 2(3):68-81

Takahashi T, Nobuo T, Masaaki L, Masaki B, Hoypku N, Hiroyuki T, Toru O. 2004. Isoliquiritigenin, a flavonoid from licorice, reduces prostaglandin E 2 and nitric oxide, causes apoptosis and suppresses aberrant crypt foci development. Cancer Sci, 95(5): 448-453.

Taleuzzaman M, Ali S, Gilani SJ, Imam SS, Hafeez A. 2015. Ultra Performance Liquid Chromatography (UPLC) - A Review, Austin J Anal Pharm Chem, 2(6): 1056.

Taufik I. 2017. Profil Metabolite Kulit Batang Artocarpus champeden Spreng Secara HPTLC Densitometry serta Hubunganna dengan Antimalaria dan Toksisitas In Vitro [Skripsi], Prodi Magister Ilmu Farmasi UNAIR: Surabaya.

Verma N, Shukla S. 2015. Impact of Various Factors Responsible for Fluctuation in Plant Secondary Metabolites, Journal of Applied Research on Medicinal and Aromatic Plants, 2(4) : 49.

Wang N, Zhiyu W, Cheng P, Jieshu Y, Jiangang S, Shouwei H, Jianping C. 2013. Dietary Compound Isoliquiritigenin Inhibits Breast Cancer Neoangiogenesis via VEGF/VEGFR-2 Signaling Pathway, Plos One, 8(7):582-601.

Wolfendera, Jean l, Guillaume M, Aurelien T, and Samuel B. 2015, Current Approaches and Challenges for the Metabolite Profiling of Complex Natural Extracts, Journal of Chromatography A, 1382: 136-164. 